\section{Database of proteinogenic amino acid reference spectra for Bismuth-cluster ToF-SIMS. II. Positive polarity}

\author{
Maciej Kawecki ${ }^{\text {a) }}$ \\ Laboratory of Nanoscale Materials Science, Empa, CH-8600 Dübendorf, Switzerland and \\ Department of Physics, University of Basel, CH-4056 Basel, Switzerland
}

Laetitia Bernard

Laboratory of Nanoscale Materials Science, Empa, CH-8600 Dübendorf, Switzerland

(Received 6 February 2018; accepted 1 May 2018; published 10 July 2018)

The number of time-of-flight secondary ion mass spectrometry studies on biological tissues and cells strongly increased since the development of primary ion sources that allow not only elemental but also molecular analysis. Still, substantial fragmentation during ionic bombardment results in a large amount of peaks, rendering data analysis complex. Complete and trustable sets of reference spectra for the main biological building blocks such as proteins, sugars, and lipids are required. Here, the authors provide a database of reference spectra for the 21 proteinogenic amino acids + glycine for bismuth instruments. Paper 1 of this two-part article series contains the negative polarity spectra and Paper II the positive polarity spectra. These reference spectra were obtained with the primary ion $\mathrm{Bi}_{3}{ }^{+}$, and spectra for the primary ions $\mathrm{Bi}_{1}{ }^{+}$and $\mathrm{Bi}_{3}{ }^{++}$are contained in the supplementary material as ASCII files. Published by the AVS.

https://doi.org/10.1116/1.5024988

Keywords: ToF-SIMS, amino acid, mass spectrometry, fragmentation

Accession \#: 01453, 01454, 01455,
01456, 01457, 01458, 01459,
01460, 01461, 01462, 01463,
01464, 01465, 01466, 01467,
01468, 01469, 01470, 01471,
01472, 01473, 01474
Technique: SIMS
Host Material: Silicon (100) wafer
Instrument: IONTOF TOF-SIMS.5
Major Species in Spectra: C, H, N,
O, S
Minor Species in Spectra: Na, K, Si
Published Spectra: 22
Spectra in Electronic Record: 66
Published Figures: 44
Spectral Category: Reference

\section{INTRODUCTION}

Partial amino acid databases for time-of-flight secondary ion mass spectrometry (ToF-SIMS) reference mass spectra have been already conducted based on the now largely replaced monoatomic argon (Ref. 1) and caesium (Ref. 2) primary ion sources. The characteristic molecular fragmentation in ToFSIMS measurements, however, depends on the primary ion impacting the surface. The current standard primary ion source is the bismuth/manganese liquid metal ion source. For bismuth and bismuth cluster primary ions, only limited lists attributing prominent peaks to specific amino acids can be found in the literature, e.g., Ref. 3. Here, we provide a database of original reference spectra for the 21 proteinogenic amino acids + glycine as well as the complete corresponding peak lists determining the fragmentation products. Fragments which uniquely represent specific amino acids are highlighted in Table 24. This Paper II of the database contains the positive polarity spectra. Negative polarity spectra are contained in Paper I (Ref. 4).

The amino acids were purchased through Sigma Aldrich (Article number: 09416, purity $>99.0 \%$ ). Each amino acid was dissolved in $\mathrm{H}_{2} \mathrm{O}$ (Sigma Aldrich, Article number: 270733) and 0.1 M solutions were so prepared. Host silicon wafers of $1 \times 1 \mathrm{~cm}$ size were first washed by submersion in subsequent ultrasound baths of $2 \times 15 \mathrm{~min}$ in acetone and $1 \times 15 \mathrm{~min}$ in ethanol, and then plasma-treated for a duration of $10 \mathrm{~min}$ to render the surface hydrophilic. The $0.1 \mathrm{M}$ amino acid solutions were subsequently drop-deposited inside a laminar flow chamber on separate silicon wafers. It was

a)Electronic mail: maciej.kawecki@empa.ch made sure that each deposited droplet is spread over the entire wafer surface before leaving the wafers in the laminar flow chamber for drying.

The spectra were obtained on a ToF-SIMS.5 instrument (IONTOF) equipped with a $25 \mathrm{keV}$ bismuth-manganese liquid metal ion gun. The instrument was operated in high current bunched mode. The primary ion dose was kept below the static limit $\left(10^{12}\right.$ ions $\left./ \mathrm{cm}^{2}\right)$ for each spectrum, and a low-energy electron flood gun was used to compensate charging effects. The raster size was $200 \times 200 \mu \mathrm{m}^{2}$ with a raster resolution of $128 \times 128$, a cycle time of $100 \mu$ s and primary ion currents of $1.54 \mathrm{pA}$ for $\mathrm{Bi}_{1}{ }^{+}, 0.37-0.4 \mathrm{pA}$ for $\mathrm{Bi}_{3}{ }^{+}$, and $0.44 \mathrm{pA}$ for $\mathrm{Bi}_{3}{ }^{++}$. Fifty scans were acquired per single mass spectrum. All presented spectra are normalized to their respective total ion count. Note that variations in mass resolution among the reference spectra (see Fig. 23) are due to crystallization geometry differences between the amino acids.

Figures and tables presented contain only attributions for the most prominent peaks. Complete peak-lists attributing fragmentation products to 50-100 main peaks for each amino acid are included in the attachment. In the figures, the full molecule is referred to with " $M$." Polymerization products are marked with "p.p.", cationization products with "c.p.," and contaminants with "c." Major peaks where a unique attribution was not possible, e.g., due to an overlap beyond mass resolution of two potential fragmentation products, are marked with "n.a." standing for not (uniquely) attributed. In the label of the supplementary material files, the Spectrum ID\# uniquely identifies each amino acid. Mass spectra acquired using $\mathrm{Bi}_{3}{ }^{+}, \mathrm{Bi}_{1}{ }^{+}$, and $\mathrm{Bi}_{3}{ }^{++}$primary ions are further identified by the file-suffix " 01, , " 02 ," and " 03 ," respectively, and peak lists in ASCII format by the file-suffix "04." 
SPECIMEN DESCRIPTION (ACCESSION \# 01453, 01454, 01455, 01456, 01457, 01458, 01459, 01460, 01461, 01462, 01463, 01464, 01465, 01466, 01467, 01468, 01469, 01470, 01471, 01472, 01473, 01474)

Host Material: Silicon (100) wafer

CAS Registry \#: 7440-21-3

Host Material Characteristics: Homogeneous; solid; single crystal; semiconductor; semiconductor

Chemical Name: L-alanine (01453), L-arginine monohydrochloride (01454), L-asparagine (01455), L-asparatic acid (01456), L-cysteine (01457), L-cystine (01458), L-glutamic acid (01459), L-glutamine (01460), glycine (01461), L-histidine monohydrochloride (01462), L-4-hydroxiproline (01463), L-isoleucine (01464), L-leucine (01465), L-lysine monohydrochloride (01466), L-methionine (01467), L-phenylalanine (01468), L-proline (01469), L-serine (01470), L-threonine (01471), L-tryptophan (01472), L-tyrosine (01473), L-valine (01474)

Source: Sigma Aldrich

Host Composition: $\mathrm{Si}$ and $\mathrm{SiO}_{2}$

Form: Crystalline powder dissolved in DI $\mathrm{H}_{2} \mathrm{O}$ and subsequently drop-deposited on the host wafer

Lot Number: L-alanine: BCBS2461V, L-arginine monohydrochloride: $1361811 \mathrm{~V}$, L-asparagine: $1371738 \mathrm{~V}$, L-asparatic acid: BCBR6815V, L-cysteine: BCBR4500V, L-cystine: BCBS1903V, L-glutamic acid: $1423805 \mathrm{~V}$, L-glutamine: BCBR7569V, glycine: $1119375 \mathrm{~V}$, L-histidine monohydrochloride: BCBS1876V, L-4hydroxiproline: BCBL2666V, L-isoleucine: $1423806 \mathrm{~V}$, L-leucine: BCBBQ9986V, L-lysine monohydrochloride: BCBN9886V, L-methionine: 1423807V, L-phenylalanine: BCBQ1493V, L-proline: BCBP4505V, L-serine: 1336081V, L-threonine: BCBD4901V, L-tryptophan: BCBR0168V, L-tyrosine: BCBR7417V, L-valine: BCBM0163V

Structure: L-alanine: $\mathrm{C}_{3} \mathrm{H}_{7} \mathrm{NO}_{2}$, L-arginine monohydrochloride: $\mathrm{C}_{6} \mathrm{H}_{14} \mathrm{~N}_{4} \mathrm{O}_{2} \cdot \mathrm{HCl}$, L-asparagine: $\mathrm{C}_{4} \mathrm{H}_{8} \mathrm{~N}_{2} \mathrm{O}_{3}$, L-asparatic acid: $\mathrm{C}_{4} \mathrm{H}_{7} \mathrm{NO}_{4}$, L-cysteine: $\mathrm{C}_{3} \mathrm{H}_{7} \mathrm{NO}_{2} \mathrm{~S}$, L-cystine: $\mathrm{C}_{6} \mathrm{H}_{12} \mathrm{~N}_{2} \mathrm{O}_{4} \mathrm{~S}_{2}$, L-glutamic acid: $\mathrm{C}_{5} \mathrm{H}_{9} \mathrm{NO}_{4}$, L-glutamine: $\mathrm{C}_{5} \mathrm{H}_{10} \mathrm{~N}_{2} \mathrm{O}_{3}$, glycine: $\mathrm{C}_{2} \mathrm{H}_{5} \mathrm{NO}_{2}$, L-histidine monohydrochloride: $\mathrm{C}_{6} \mathrm{H}_{9} \mathrm{~N}_{3} \mathrm{O}_{2} \cdot \mathrm{HCl}$, L-4hydroxiproline: $\mathrm{C}_{5} \mathrm{H}_{9} \mathrm{NO}_{3}$, L-isoleucine: $\mathrm{C}_{6} \mathrm{H}_{13} \mathrm{NO}_{2}$, L-leucine: $\mathrm{C}_{6} \mathrm{H}_{13} \mathrm{NO}_{2}$, L-lysine monohydrochloride: $\mathrm{C}_{6} \mathrm{H}_{14} \mathrm{~N}_{2} \mathrm{O}_{2} \cdot \mathrm{HCl}$, L-methionine: $\mathrm{C}_{5} \mathrm{H}_{11} \mathrm{NO}_{2} \mathrm{~S}$, L-phenylalanine: $\mathrm{C}_{9} \mathrm{H}_{11} \mathrm{NO}_{2}$, L-proline: $\mathrm{C}_{5} \mathrm{H}_{9} \mathrm{NO}_{2}$, L-serine: $\mathrm{C}_{3} \mathrm{H}_{7} \mathrm{NO}_{3}$, L-threonine: $\mathrm{C}_{4} \mathrm{H}_{9} \mathrm{NO}_{3}$, L-tryptophan: $\mathrm{C}_{11} \mathrm{H}_{12} \mathrm{~N}_{2} \mathrm{O}_{2}$, L-tyrosine: $\mathrm{C}_{9} \mathrm{H}_{11} \mathrm{NO}_{3}$, L-valine: $\mathrm{C}_{5} \mathrm{H}_{11} \mathrm{NO}_{2}$

History and Significance: The proteinogenic amino acids are the building blocks of all proteins in the known life forms. Complete spectra databases of organic molecules facilitate the interpretation of ToF-SIMS data from biological specimen. However, it should be kept in mind that in biological specimens amino acids are incorporated into larger molecules (proteins, peptidoglycans, etc.) which affects the fragmentation patterns.

As Received Condition: Samples received in powder form

Analyzed Region: Regions of $200 \times 200 \mu \mathrm{m}^{2}$ on maximally homogeneous specimen sites

Ex Situ Preparation/Mounting: 0.1 M amino acid solutions were prepared in $\mathrm{H}_{2} \mathrm{O}$ and drop-deposited on separate $1 \times 1 \mathrm{~cm}$ large plasma-treated $\mathrm{Si}$-Wafers in a laminar flow chamber.
In Situ Preparation: None

Charge Control: Low energy electrons

Temp. During Analysis: $300 \mathrm{~K}$

Pressure During Analysis: $5.6 \times 10^{-8} \mathrm{~Pa}$

Preanalysis Beam Exposure: None

INSTRUMENT CONFIGURATION

Manufacturer and Model: IONTOF TOF-SIMS.5

Analyzer Type: Time-of-flight

Detector Type: Microchannel plate

Experiment Type: Mass spectra

Sample Rotation: None

Oxygen Flood Source: None

Other Flood Source: Low energy electrons

Energy Acceptance Window: $14.3 \mathrm{eV}$

Postacceleration Voltage: $10000 \mathrm{eV}$

Sample Bias: $0 \mathrm{eV}$

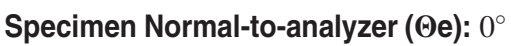

\section{- Ion Sources}

Ion Source 1 of 1

Purpose of this Ion Source: Analysis beam

Ion Source Manufacturer: IONTOF (Münster, Germany)

Ion Source Model: $25 \mathrm{keV} \mathrm{Bi/Mn} \mathrm{cluster} \mathrm{source}$

Beam Mass Filter: Electrodynamic mass filter

Beam Species and Charge State: $\mathrm{Bi}_{3}{ }^{+}$

Beam Gating Used: None

Beam Voltage: $25000 \mathrm{eV}$

Net Beam Voltage (impact voltage): $25000 \mathrm{eV}$

Ion Pulse Width: $29.3 \mathrm{~ns}$

Ion Pulse Rate: $10 \mathrm{kHz}$

Pulsed Beam Current: 0.00037-0.0004 nA

Current Measurement Method: Faraday cup

Beam Raster Size: $200 \times 200 \mu \mathrm{m}^{2}$

Beam Incident Angle: $45^{\circ}$

Source-to-Analyzer Angle: $45^{\circ}$

\section{ACKNOWLEDGMENTS}

The authors are thankful for the funding received from the Swiss National Science Foundation through the Grant No. CR23I2-162828.

\section{REFERENCES}

1. Preinstalled database on IONTOF GmbH instruments.

2. N. T. Samuel, M. S. Wagner, K. D. Dornfeld, and D. G. Castner, Surf. Sci. Spectra 8, 163 (2001).

3. M. Urbini, V. Piteto, F. de Notaristefani, F. Scaldaferri, A. Gasbarrini, and L. Tortora, Anal. Bioanal. Chem. 409, 6097 (2017).

4. M. Kawecki and L. Bernard, Surf. Sci. Spectra 25, 015001 (2018). 


\begin{tabular}{lccc}
\hline & \multicolumn{3}{c}{ ALANINE } \\
\hline Spectrum ID \# & Mass (Da) & Species & Peak Assignment \\
\hline $\mathbf{0 1 4 5 3}$ & 44.0495 & $\mathrm{C}_{2} \mathrm{H}_{6} \mathrm{~N}^{+}$ & Alanine \\
$\ldots$ & 55.0178 & $\mathrm{C}_{3} \mathrm{H}_{3} \mathrm{O}^{+}$ & $\ldots$ \\
$\ldots$ & 56.0495 & $\mathrm{C}_{3} \mathrm{H}_{6} \mathrm{~N}^{+}$ & $\ldots$ \\
$\ldots$ & 70.0287 & $\mathrm{C}_{3} \mathrm{H}_{4} \mathrm{NO}^{+}$ & $\ldots$ \\
$\ldots$ & 74.0600 & $\mathrm{C}_{3} \mathrm{H}_{8} \mathrm{NO}^{+}$ & $\ldots$ \\
$\ldots$ & 90.0550 & $\mathrm{C}_{3} \mathrm{H}_{8} \mathrm{NO}_{2}^{+}$ & $\ldots$ \\
\hline
\end{tabular}

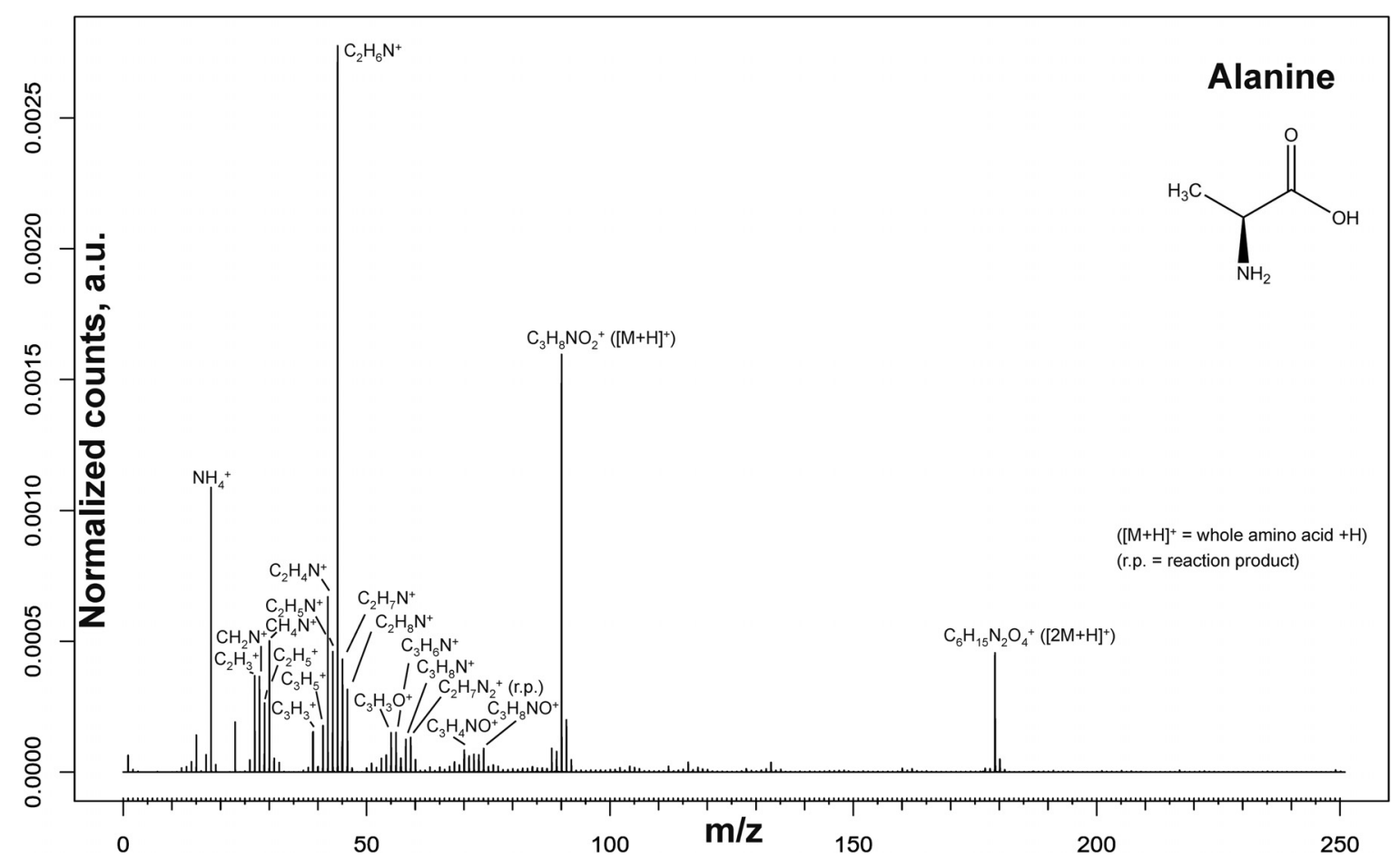

\begin{tabular}{rc}
\hline Accession \# & 01453-01, 01453-02, 01453-03, 01453-04 \\
\hline Host Material & Silicon wafer \\
Technique & SIMS \\
Secondary Source Polarity & Positive \\
Mass Range Minimum & $0 \mathrm{Da}$ \\
Mass Range Maximum & $250 \mathrm{Da}$ \\
Species Used for Mass Calibration & $\mathrm{CH}_{3}{ }^{+}, \mathrm{NH}_{3}{ }^{+}, \mathrm{NH}_{4}^{+}, \mathrm{CH}_{4} \mathrm{~N}^{+}, \mathrm{C}_{3} \mathrm{H}_{7} \mathrm{NO}_{2}{ }^{+}$ \\
Primary lon Dose & $5.11 \times 10^{11} \mathrm{~cm}^{-2}$ \\
Primary Species & $\mathrm{Bi}_{3}{ }^{+}$ \\
Primary lon Pulse Width & $29.3 \mathrm{~ns}^{-2}$ \\
Pulsed Beam Current & $0.0004 \mathrm{nA}^{2}$ \\
Beam Raster Size & $200 \times 200 \mu \mathrm{m}^{2}$ \\
\hline
\end{tabular}




\begin{tabular}{lccc}
\hline & \multicolumn{2}{c}{ ARGININE (HYDROCHLORIDE) } & \\
\hline Spectrum ID \# & Mass (Da) & Species & Peak Assignment \\
\hline $\mathbf{0 1 4 5 4}$ & 60.0556 & $\mathrm{CH}_{6} \mathrm{~N}_{3}{ }^{+}$ & Arginine \\
$\ldots$ & 70.0777 & $\mathrm{C}_{5} \mathrm{H}_{10}{ }^{+}$ & $\ldots$ \\
$\ldots$ & 73.0634 & $\mathrm{C}_{2} \mathrm{H}_{7} \mathrm{~N}_{3}{ }^{+}$ & $\ldots$ \\
$\ldots$ & 87.0791 & $\mathrm{C}_{3} \mathrm{H}_{9} \mathrm{~N}_{3}{ }^{+}$ & $\ldots$ \\
$\ldots$ & 100.0869 & $\mathrm{C}_{4} \mathrm{H}_{10} \mathrm{~N}_{3}^{+}$ & $\ldots$ \\
$\ldots$ & 112.0869 & $\mathrm{C}_{5} \mathrm{H}_{10} \mathrm{~N}_{3}{ }^{+}$ & $\ldots$ \\
$\ldots$ & 130.1213 & $\mathrm{C}_{5} \mathrm{H}_{14} \mathrm{~N}_{4}{ }^{+}$ & $\ldots$ \\
$\ldots$ & 160.1081 & $\mathrm{C}_{6} \mathrm{H}_{14} \mathrm{~N}_{3} \mathrm{O}_{2}{ }^{+}$ & $\ldots$ \\
$\ldots$ & 175.1190 & $\mathrm{C}_{6} \mathrm{H}_{15} \mathrm{~N}_{4} \mathrm{O}_{2}{ }^{+}$ & $\ldots$ \\
\hline
\end{tabular}

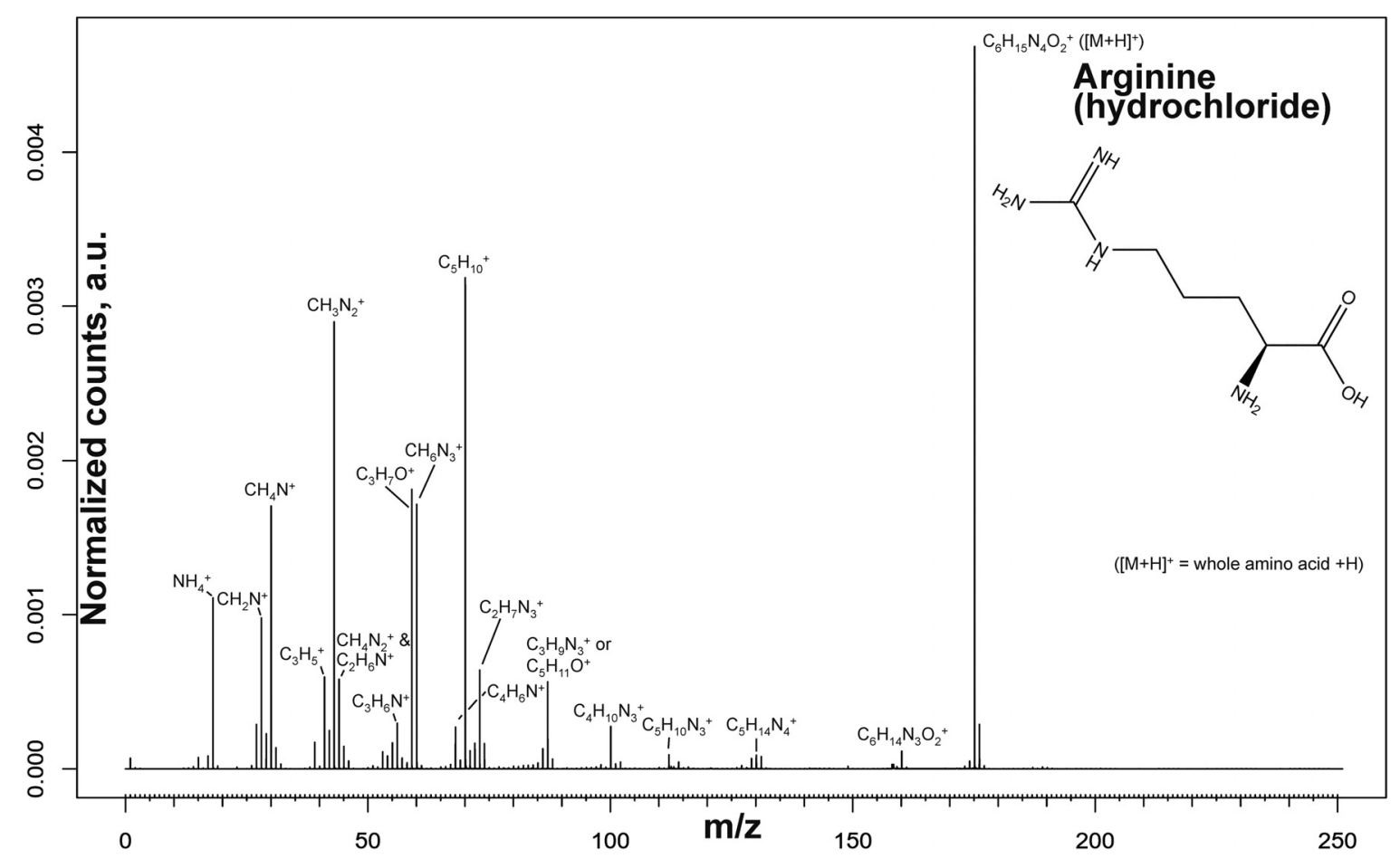

\begin{tabular}{rc}
\hline Accession \# & 01454-01, 01454-02, 01454-03, 01454-04 \\
\hline Host Material & Silicon wafer \\
Technique & SIMS \\
Secondary Source Polarity & Positive \\
Mass Range Minimum & $0 \mathrm{Da}$ \\
Mass Range Maximum & $250 \mathrm{Da}$ \\
Species Used for Mass Calibration & $\mathrm{CH}_{3}{ }^{+}, \mathrm{NH}_{3}{ }^{+}, \mathrm{NH}_{4}^{+}, \mathrm{CH}_{2} \mathrm{~N}^{+}, \mathrm{C}_{6} \mathrm{H}_{14} \mathrm{~N}_{4} \mathrm{O}_{2}{ }^{+}$ \\
Primary lon Dose & $4.73 \times 10^{11} \mathrm{~cm}^{-2}$ \\
Primary Species & $\mathrm{Bi}_{3}{ }^{+}$ \\
Primary lon Pulse Width & $29.3 \mathrm{~ns}^{-}$ \\
Pulsed Beam Current & $0.00037 \mathrm{nA}^{2}$ \\
Beam Raster Size & $200 \times 200 \mu \mathrm{m}^{2}$ \\
\hline
\end{tabular}




\begin{tabular}{lccc}
\hline \multicolumn{3}{c}{ ASPARAGINE } \\
\hline Spectrum ID \# & Mass (Da) & Species & Peak Assignment \\
\hline $\mathbf{0 1 4 5 5}$ & 70.0287 & $\mathrm{C}_{3} \mathrm{H}_{4} \mathrm{NO}^{+}$ & Asparagine \\
$\ldots$ & 74.0237 & $\mathrm{C}_{2} \mathrm{H}_{4} \mathrm{NO}_{2}{ }^{+}$ & $\ldots$ \\
$\ldots$ & 87.0553 & $\mathrm{C}_{3} \mathrm{H}_{7} \mathrm{~N}_{2} \mathrm{O}^{+}$ & $\ldots$ \\
$\ldots$ & 89.0709 & $\mathrm{C}_{3} \mathrm{H}_{9} \mathrm{~N}_{2} \mathrm{O}^{+}$ & $\ldots$ \\
$\ldots$ & 116.0342 & $\mathrm{C}_{4} \mathrm{H}_{6} \mathrm{NO}_{3}{ }^{+}$ & $\ldots$ \\
$\ldots$ & 133.0608 & $\mathrm{C}_{4} \mathrm{H}_{9} \mathrm{~N}_{2} \mathrm{O}_{3}{ }^{+}$ & $\ldots$ \\
\hline
\end{tabular}

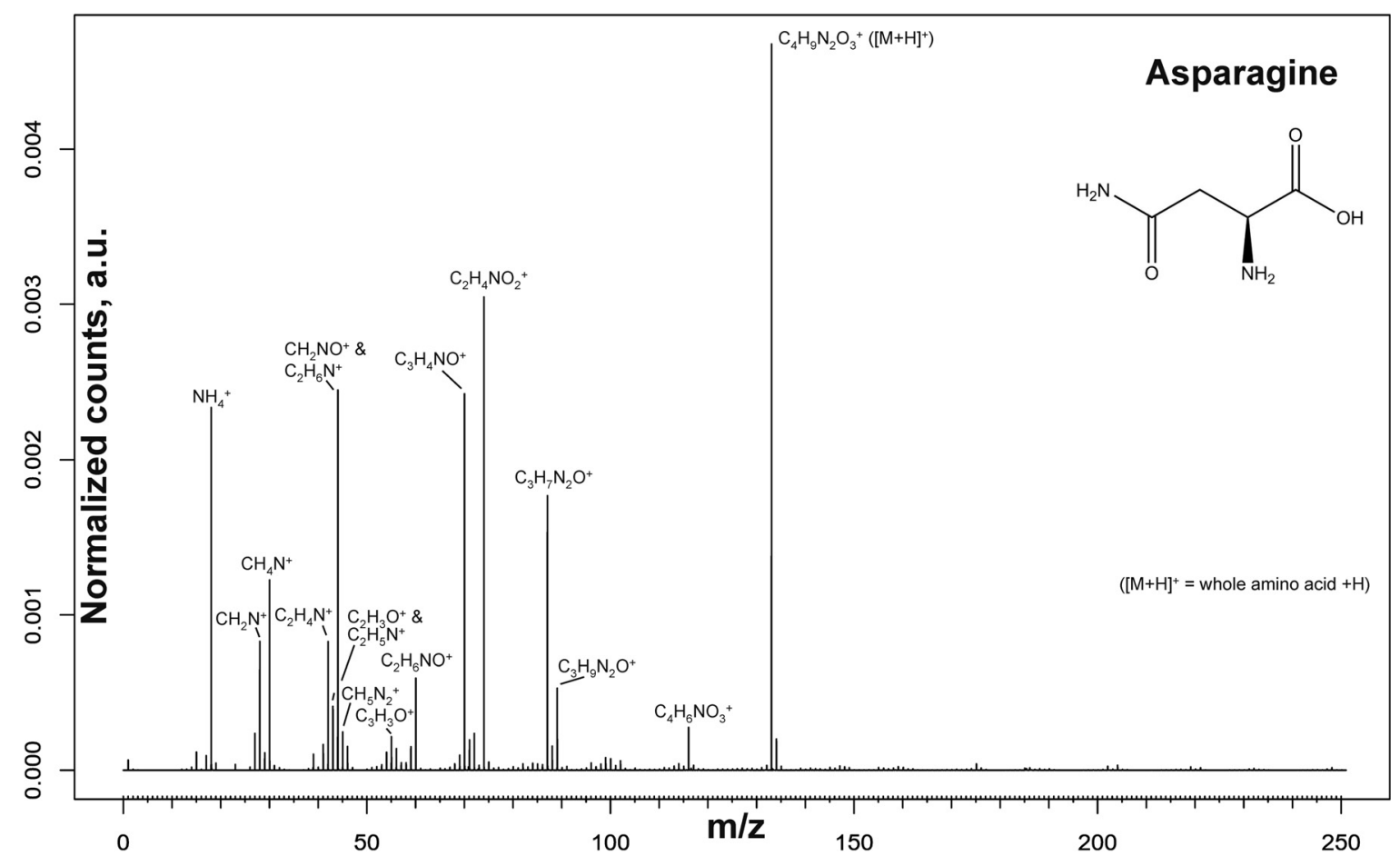

Accession \#

01455-01, 01455-02, 01455-03, 01455-04

Host Material

- Technique

Secondary Source Polarity

Mass Range Minimum

Mass Range Maximum

Species Used for Mass Calibration

Primary lon Dose

Primary Species

Primary lon Pulse Width

Pulsed Beam Current

Beam Raster Size
Silicon wafer

SIMS

Positive

$0 \mathrm{Da}$

$250 \mathrm{Da}$

$\mathrm{CH}_{3}{ }^{+}, \mathrm{NH}_{3}{ }^{+}, \mathrm{CH}_{2} \mathrm{~N}^{+}, \mathrm{C}_{4} \mathrm{H}_{10} \mathrm{~N}_{2} \mathrm{O}_{3}{ }^{+}$

$4.73 \times 10^{11} \mathrm{~cm}^{-2}$

$\mathrm{Bi}_{3}{ }^{+}$

$29.3 \mathrm{~ns}$

$0.00037 \mathrm{nA}$

$200 \times 200 \mu \mathrm{m}^{2}$ 


\begin{tabular}{lccc}
\hline \multicolumn{3}{c}{ ASPARATIC ACID } \\
\hline Spectrum ID \# & Mass (Da) & Species & Peak Assignment \\
\hline $\mathbf{0 1 4 5 6}$ & 70.0287 & $\mathrm{C}_{3} \mathrm{H}_{4} \mathrm{NO}^{+}$ & Asparatic acid \\
$\ldots$ & 74.0237 & $\mathrm{C}_{2} \mathrm{H}_{4} \mathrm{NO}_{2}{ }^{+}$ & $\ldots$ \\
$\ldots$ & 88.0393 & $\mathrm{C}_{3} \mathrm{H}_{6} \mathrm{NO}_{2}{ }^{+}$ & $\ldots$ \\
$\ldots$ & 90.0550 & $\mathrm{C}_{3} \mathrm{H}_{8} \mathrm{NO}_{2}{ }^{+}$ & $\ldots$ \\
$\ldots$ & 134.0448 & $\mathrm{C}_{4} \mathrm{H}_{8} \mathrm{NO}_{4}^{+}$ & $\ldots$ \\
\hline
\end{tabular}

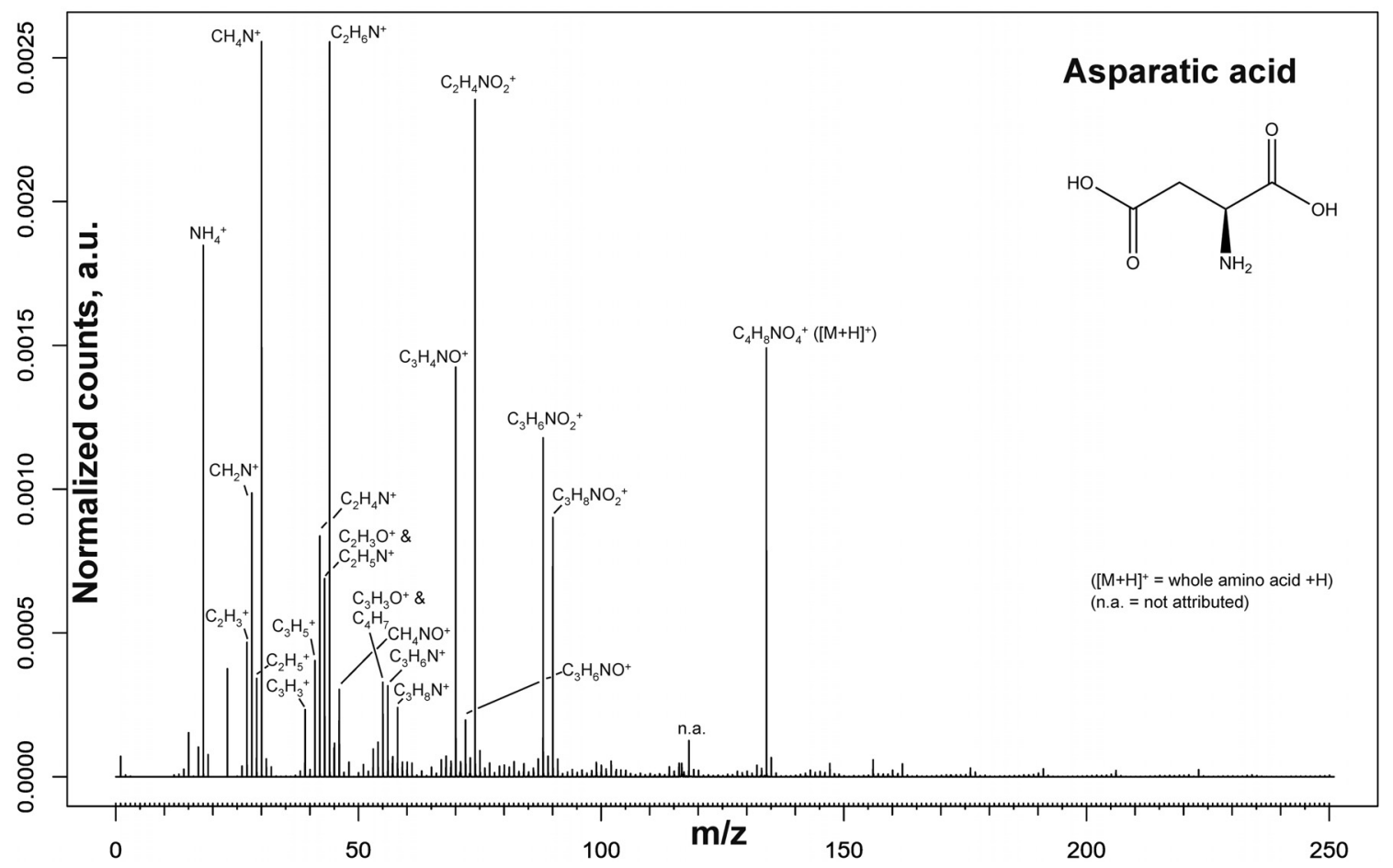

\begin{tabular}{rc}
\hline Accession \# & 01456-01, 01456-02, 01456-03, 01456-04 \\
\hline Host Material & Silicon wafer \\
$\mathbf{\text { Technique }}$ & $\mathrm{SIMS}$ \\
Secondary Source Polarity & Positive \\
Mass Range Minimum & $0 \mathrm{Da}$ \\
Mass Range Maximum & $250 \mathrm{Da}$ \\
Species Used for Mass Calibration & $\mathrm{CH}_{3}{ }^{+}, \mathrm{NH}_{3}{ }^{+}, \mathrm{CH}_{2} \mathrm{~N}^{+}, \mathrm{C}_{4} \mathrm{H}_{9} \mathrm{NO}_{4}{ }^{+}$ \\
Primary lon Dose & $4.73 \times 10^{11} \mathrm{~cm}^{-2}$ \\
Primary Species & $\mathrm{Bi}_{3}{ }^{+}$ \\
Primary lon Pulse Width & $29.3 \mathrm{~ns}^{-}$ \\
Pulsed Beam Current & $0.00037 \mathrm{nA}^{2}$ \\
Beam Raster Size & $200 \times 200 \mu \mathrm{m}^{2}$ \\
\hline
\end{tabular}




\begin{tabular}{lccc}
\hline & & CYSTEINE & \\
\hline Spectrum ID \# & Mass (Da) & Species & Peak Assignment \\
\hline $\mathbf{0 1 4 5 7}$ & 31.9715 & $\mathrm{~S}^{+}$ & Cysteine \\
$\ldots$ & 34.9950 & $\mathrm{H}_{3} \mathrm{~S}^{+}$ & $\ldots$ \\
$\ldots$ & 44.9793 & $\mathrm{CHS}^{+}$ & $\ldots$ \\
$\ldots$ & 46.9950 & $\mathrm{CH}_{3} \mathrm{~S}^{+}$ & $\ldots$ \\
$\ldots$ & 58.9950 & $\mathrm{C}_{2} \mathrm{H}_{3} \mathrm{~S}^{+}$ & $\ldots$ \\
$\ldots$ & 75.0137 & $\mathrm{C}_{2} \mathrm{H}_{5} \mathrm{NS}^{+}$ & $\ldots$ \\
$\ldots$ & 76.0215 & $\mathrm{C}_{2} \mathrm{H}_{6} \mathrm{NS}^{+}$ & $\ldots$ \\
$\ldots$ & 86.9899 & $\mathrm{C}_{3} \mathrm{H}_{3} \mathrm{OS}^{+}$ & $\ldots$ \\
$\ldots$ & 88.0393 & $\mathrm{C}_{3} \mathrm{H}_{6} \mathrm{NO}_{2}{ }^{+}$ & $\ldots$ \\
$\ldots$ & 90.0550 & $\mathrm{C}_{3} \mathrm{H}_{8} \mathrm{NO}_{2}$ & $\ldots$ \\
$\ldots$ & 105.0005 & $\mathrm{C}_{3} \mathrm{H}_{5} \mathrm{O}_{2} \mathrm{~S}^{+}$ & $\ldots$ \\
\hline
\end{tabular}

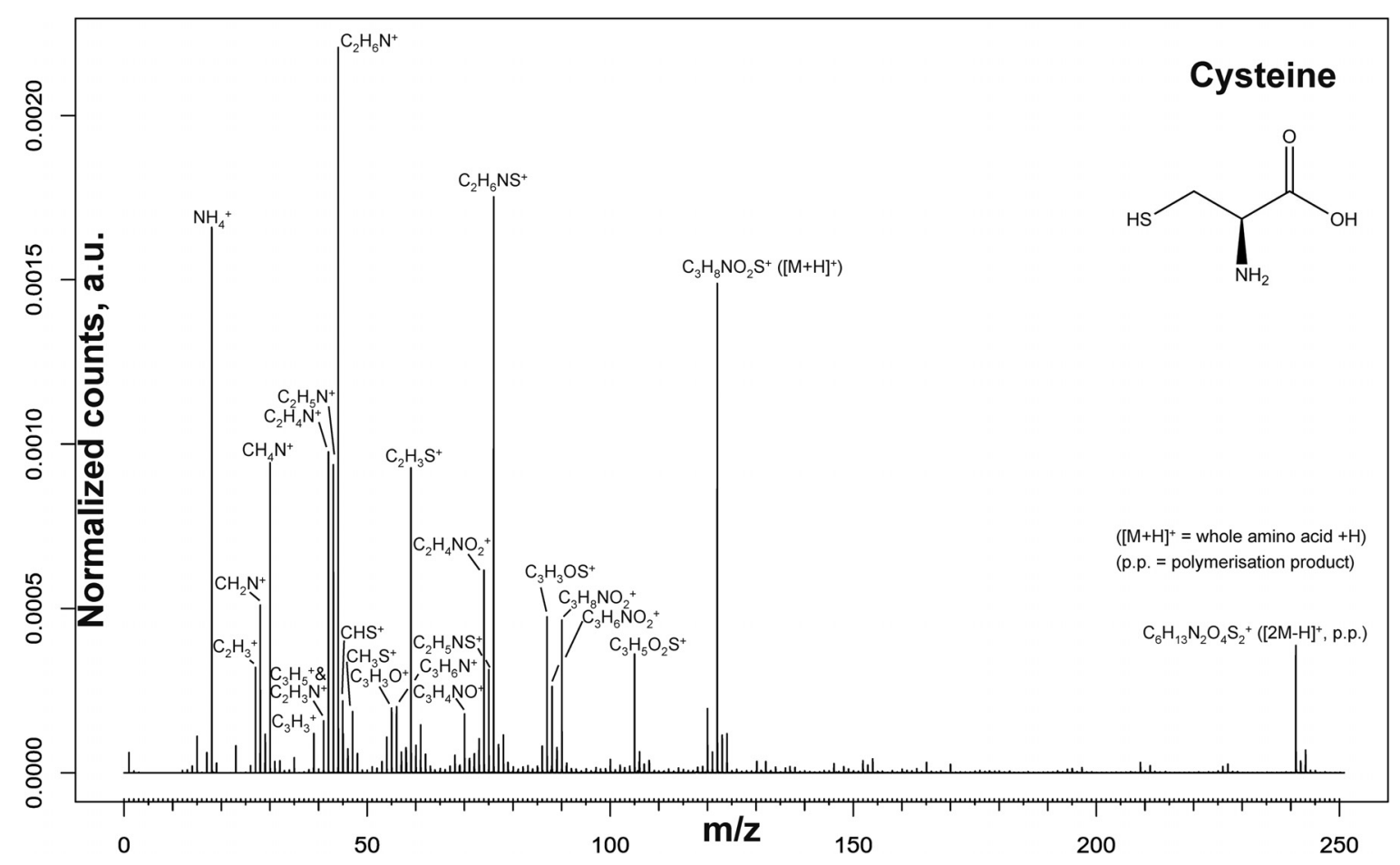

\begin{tabular}{rc}
\hline Accession \# & 01457-01, 01457-02, 01457-03, 01457-04 \\
\hline Host Material & Silicon wafer \\
$\mathbf{1}$ Technique & SIMS \\
Secondary Source Polarity & Positive \\
Mass Range Minimum & $0 \mathrm{Da}$ \\
Mass Range Maximum & $250 \mathrm{Da}$ \\
Species Used for Mass Calibration & $\mathrm{CH}_{3}{ }^{+}, \mathrm{NH}_{3}{ }^{+}, \mathrm{CH}_{2} \mathrm{~N}^{+}, \mathrm{C}_{3} \mathrm{H}_{7} \mathrm{SNO}_{2}{ }^{+}$ \\
Primary lon Dose & $4.73 \times 10^{11} \mathrm{~cm}^{-2}$ \\
Primary Species & $\mathrm{Bi}_{3}{ }^{+}$ \\
Primary lon Pulse Width & $29.3 \mathrm{~ns}^{-}$ \\
Pulsed Beam Current & $0.00037 \mathrm{nA}^{2}$ \\
Beam Raster Size & $200 \times 200 \mu \mathrm{m}^{2}$ \\
\hline
\end{tabular}




\begin{tabular}{lccc}
\hline & \multicolumn{3}{c}{ CYSTINE } \\
\hline Spectrum ID \# & Mass (Da) & Species & Peak Assignment \\
\hline $\mathbf{0 1 4 5 8}$ & 63.9436 & $\mathrm{~S}_{2}{ }^{+}$ & Cystine \\
$\ldots$ & 58.9950 & $\mathrm{C}_{2} \mathrm{H}_{3} \mathrm{~S}^{+}$ & $\ldots$ \\
$\ldots$ & 76.0215 & $\mathrm{C}_{2} \mathrm{H}_{6} \mathrm{NS}^{+}$ & $\ldots$ \\
$\ldots$ & 120.0114 & $\mathrm{C}_{3} \mathrm{H}_{6} \mathrm{NO}_{2} \mathrm{~S}^{+}$ & $\ldots$ \\
$\ldots$ & 122.0270 & $\mathrm{C}_{3} \mathrm{H}_{8} \mathrm{NO}_{2} \mathrm{~S}^{+}$ & $\ldots$ \\
$\ldots$ & 151.9834 & $\mathrm{C}_{3} \mathrm{H}_{6} \mathrm{NO}_{2} \mathrm{~S}_{2}^{+}$ & $\ldots$ \\
$\ldots$ & 195.0256 & $\mathrm{C}_{5} \mathrm{H}_{11} \mathrm{~N}_{2} \mathrm{O}_{2} \mathrm{~S}_{2}^{+}$ & $\ldots$ \\
$\ldots$ & 207.0256 & $\mathrm{C}_{6} \mathrm{H}_{11} \mathrm{~N}_{2} \mathrm{O}_{2} \mathrm{~S}_{2}{ }^{+}$ & $\ldots$ \\
$\ldots$ & 241.0311 & $\mathrm{C}_{6} \mathrm{H}_{13} \mathrm{~N}_{2} \mathrm{O}_{4} \mathrm{~S}_{2}{ }^{+}$ & $\ldots$ \\
\hline
\end{tabular}

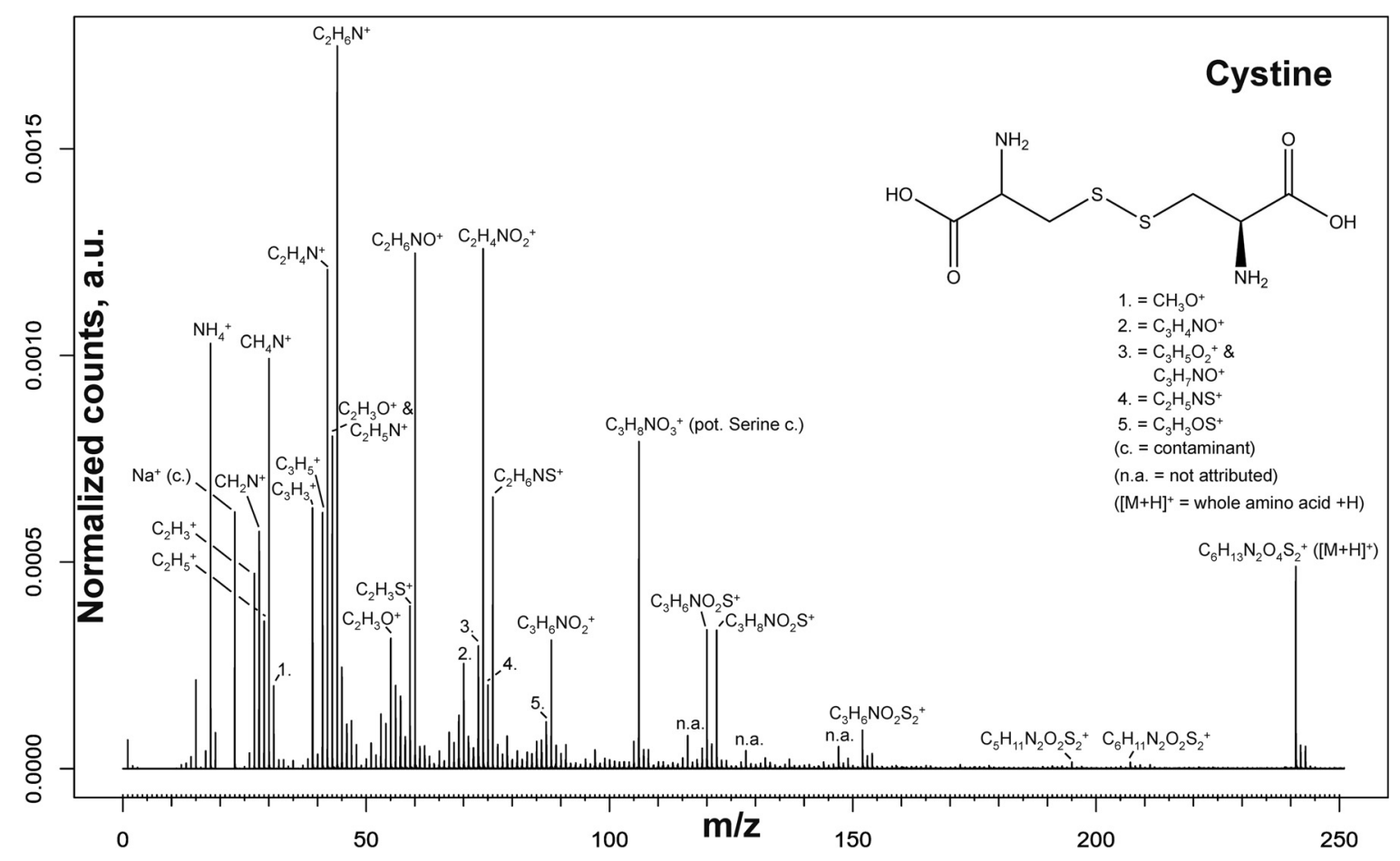

\begin{tabular}{rc}
\hline Accession \# & 01458-01, 01458-02, 01458-03, 01458-04 \\
\hline Host Material \\
$\mathbf{\text { Technique }}$ & Silicon wafer \\
Secondary Source Polarity & $\mathrm{SIMS}$ \\
Mass Range Minimum & Positive \\
Mass Range Maximum & $0 \mathrm{Da}$ \\
Primary lon Dose & $250 \mathrm{Da}$ \\
Primary Species & $\mathrm{CH}_{3}{ }^{+}, \mathrm{NH}_{3}{ }^{+}, \mathrm{NH}_{4}^{+}, \mathrm{CH}_{2} \mathrm{~N}^{+}, \mathrm{C}_{6} \mathrm{H}_{14} \mathrm{~N}_{2} \mathrm{O}_{4} \mathrm{~S}_{2}{ }^{+}$ \\
Species Used for Mass Calibration & $4.73 \times 10^{11} \mathrm{~cm}^{-2}$ \\
Primary lon Pulse Width & $\mathrm{Bi}_{3}{ }^{+}$ \\
Pulsed Beam Current & $29.3 \mathrm{~ns}$ \\
Beam Raster Size & $0.00037 \mathrm{nA}$ \\
Comment & $200 \times 200 \mu \mathrm{m}^{2}$ \\
& Region of interest, defined by a minimum threshold of the $\mathrm{C}_{2} \mathrm{H}_{4} \mathrm{NO}_{2}{ }^{+}$ \\
signal, was applied to eliminate the substrate signal.
\end{tabular}




\begin{tabular}{lccc}
\hline \multicolumn{3}{c}{ GLUTAMIC ACID } \\
\hline Spectrum ID \# & Mass (Da) & Species & Peak Assignment \\
\hline $\mathbf{0 1 4 5 9}$ & 84.0444 & $\mathrm{C}_{4} \mathrm{H}_{6} \mathrm{NO}^{+}$ & Glutamic acid \\
$\ldots$ & 84.0808 & $\mathrm{C}_{5} \mathrm{H}_{10} \mathrm{~N}^{+}$ & $\ldots$ \\
$\ldots$ & 91.0628 & $\mathrm{C}_{3} \mathrm{H}_{9} \mathrm{NO}_{2}{ }^{+}$ & $\ldots$ \\
$\ldots$ & 102.0550 & $\mathrm{C}_{4} \mathrm{H}_{8} \mathrm{NO}_{2}{ }^{+}$ & $\ldots$ \\
$\ldots$ & 148.0604 & $\mathrm{C}_{5} \mathrm{H}_{10} \mathrm{NO}_{4}^{+}$ & $\ldots$ \\
\hline
\end{tabular}

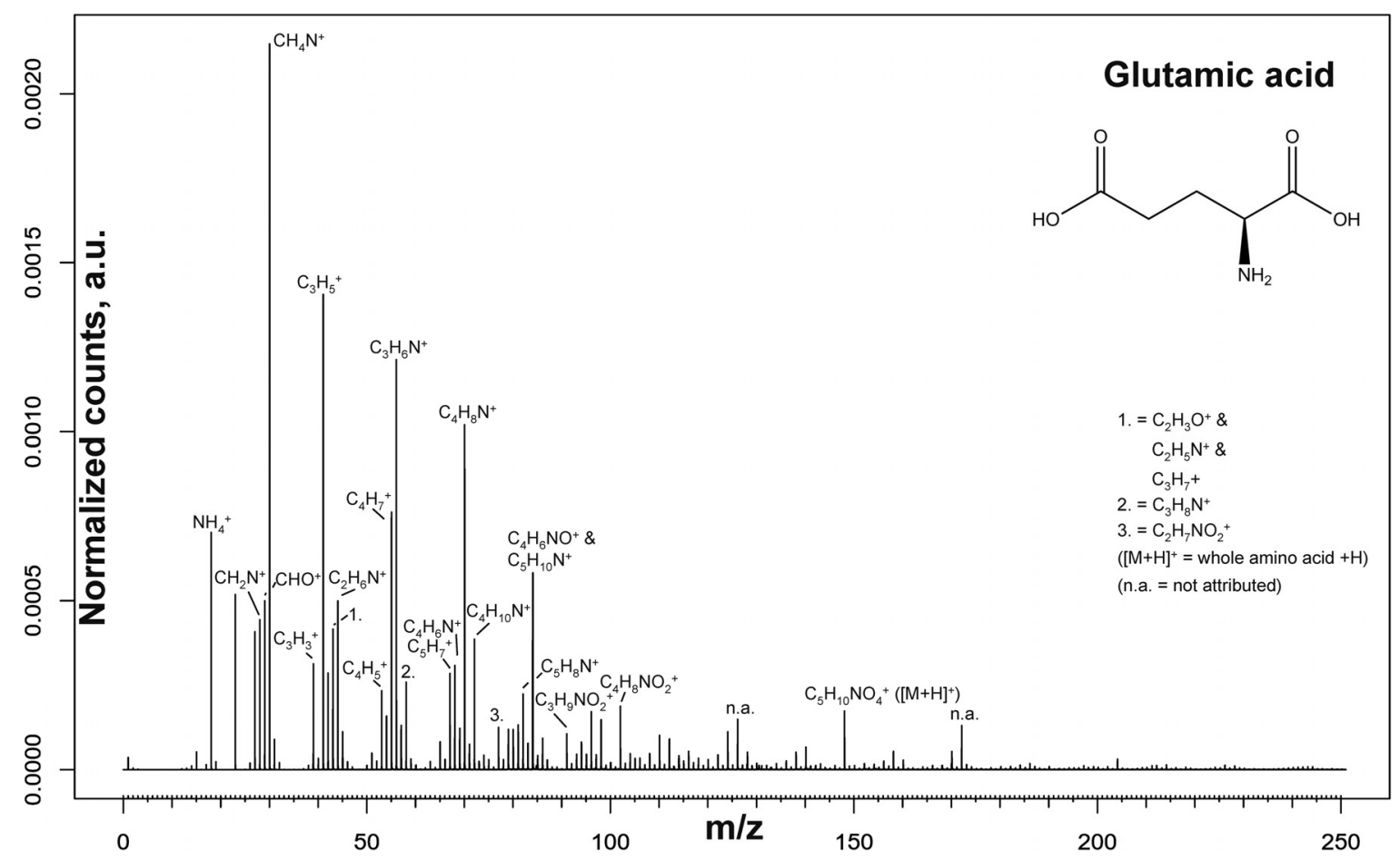

Accession \#

01459-01, 01459-02, 01459-03, 01459-04

Host Material

- Technique

Secondary Source Polarity

Mass Range Minimum

Mass Range Maximum

Species Used for Mass Calibration

Primary lon Dose

Primary Species

Primary lon Pulse Width

Pulsed Beam Current

Beam Raster Size
Silicon wafer

SIMS

Positive

$0 \mathrm{Da}$

$250 \mathrm{Da}$

$\mathrm{CH}_{3}{ }^{+}, \mathrm{NH}_{3}{ }^{+}, \mathrm{NH}_{4}^{+}, \mathrm{CH}_{2} \mathrm{~N}^{+}, \mathrm{C}_{5} \mathrm{H}_{10} \mathrm{NO}_{4}^{+}$

$4.73 \times 10^{11} \mathrm{~cm}^{-2}$

$\mathrm{Bi}_{3}{ }^{+}$

$29.3 \mathrm{~ns}$

$0.00037 \mathrm{nA}$

$200 \times 200 \mu \mathrm{m}^{2}$ 


\begin{tabular}{lccc}
\hline \multicolumn{3}{c}{ GLUTAMINE } & \\
\hline Spectrum ID \# & Mass (Da) & Species & Peak Assignment \\
\hline $\mathbf{0 1 4 6 0}$ & 84.0444 & $\mathrm{C}_{4} \mathrm{H}_{6} \mathrm{NO}^{+}$ & Glutamine \\
$\ldots$ & 86.0600 & $\mathrm{C}_{4} \mathrm{H}_{8} \mathrm{NO}^{+}$ & $\ldots$ \\
$\ldots$ & 101.0709 & $\mathrm{C}_{4} \mathrm{H}_{9} \mathrm{~N}_{2} \mathrm{O}^{+}$ & $\ldots$ \\
$\ldots$ & 102.0550 & $\mathrm{C}_{4} \mathrm{H}_{8} \mathrm{NO}_{2}$ & $\ldots$ \\
$\ldots$ & 103.0866 & $\mathrm{C}_{4} \mathrm{H}_{11} \mathrm{~N}_{2} \mathrm{O}^{+}$ & $\ldots$ \\
$\ldots$ & 114.0550 & $\mathrm{C}_{5} \mathrm{H}_{8} \mathrm{NO}_{2}$ & $\ldots$ \\
$\ldots$ & 130.0499 & $\mathrm{C}_{5} \mathrm{H}_{8} \mathrm{NO}_{3}{ }^{+}$ & $\ldots$ \\
$\ldots$ & 147.0764 & $\mathrm{C}_{5} \mathrm{H}_{11} \mathrm{~N}_{2} \mathrm{O}_{3}{ }^{+}$ & $\ldots$ \\
\hline
\end{tabular}

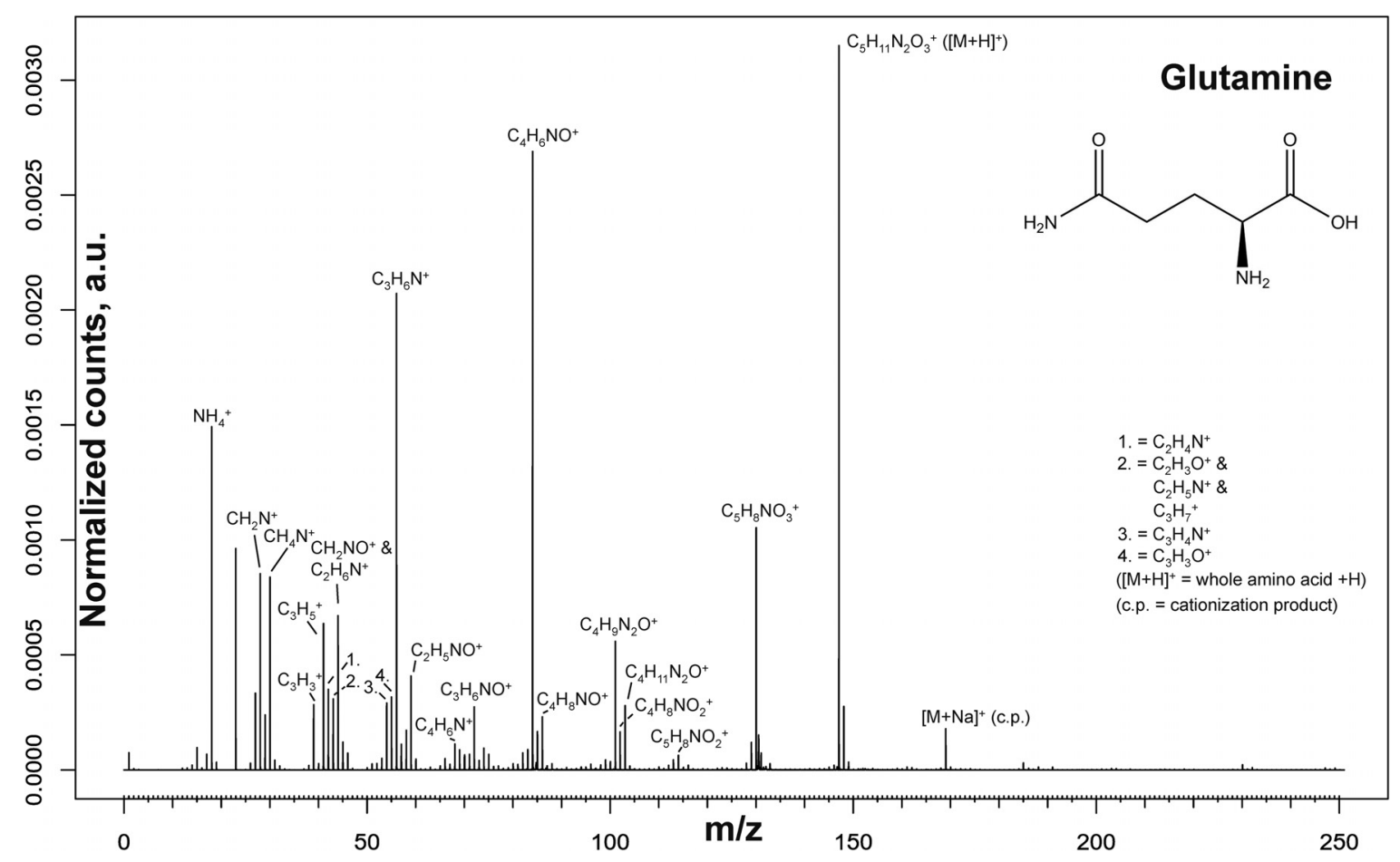

\begin{tabular}{rc}
\hline Accession \# & 01460-01, 01460-02, 01460-03, 01460-04 \\
\hline Host Material & Silicon wafer \\
Technique & SIMS \\
Secondary Source Polarity & Positive \\
Mass Range Minimum & $0 \mathrm{Da}$ \\
Mass Range Maximum & $250 \mathrm{Da}$ \\
Species Used for Mass Calibration & $\mathrm{CH}_{3}{ }^{+}, \mathrm{NH}_{3}{ }^{+}, \mathrm{CH}_{2} \mathrm{~N}^{+}, \mathrm{C}_{5} \mathrm{H}_{12} \mathrm{~N}_{2} \mathrm{O}_{3}{ }^{+}$ \\
Primary lon Dose & $4.73 \times 10^{11} \mathrm{~cm}^{-2}$ \\
Primary Species & $\mathrm{Bi}_{3}{ }^{+}$ \\
Primary lon Pulse Width & $29.3 \mathrm{~ns}^{-}$ \\
Pulsed Beam Current & $0.00037 \mathrm{nA}^{2}$ \\
Beam Raster Size & $200 \times 200 \mu \mathrm{m}^{2}$ \\
\hline
\end{tabular}




\begin{tabular}{lccc}
\hline \multicolumn{3}{c}{ GLYCINE } \\
\hline Spectrum ID \# & Mass (Da) & Species & Peak Assignment \\
\hline $\mathbf{0 1 4 6 1}$ & 43.0718 & $\mathrm{C}_{2} \mathrm{H}_{3} \mathrm{O}^{+}$ & Glycine \\
$\ldots$ & 60.0444 & $\mathrm{C}_{2} \mathrm{H}_{6} \mathrm{NO}^{+}$ & $\cdots$ \\
$\ldots$ & 76.0393 & $\mathrm{C}_{2} \mathrm{H}_{6} \mathrm{NO}_{2}{ }^{+}$ & $\ldots$ \\
\hline
\end{tabular}

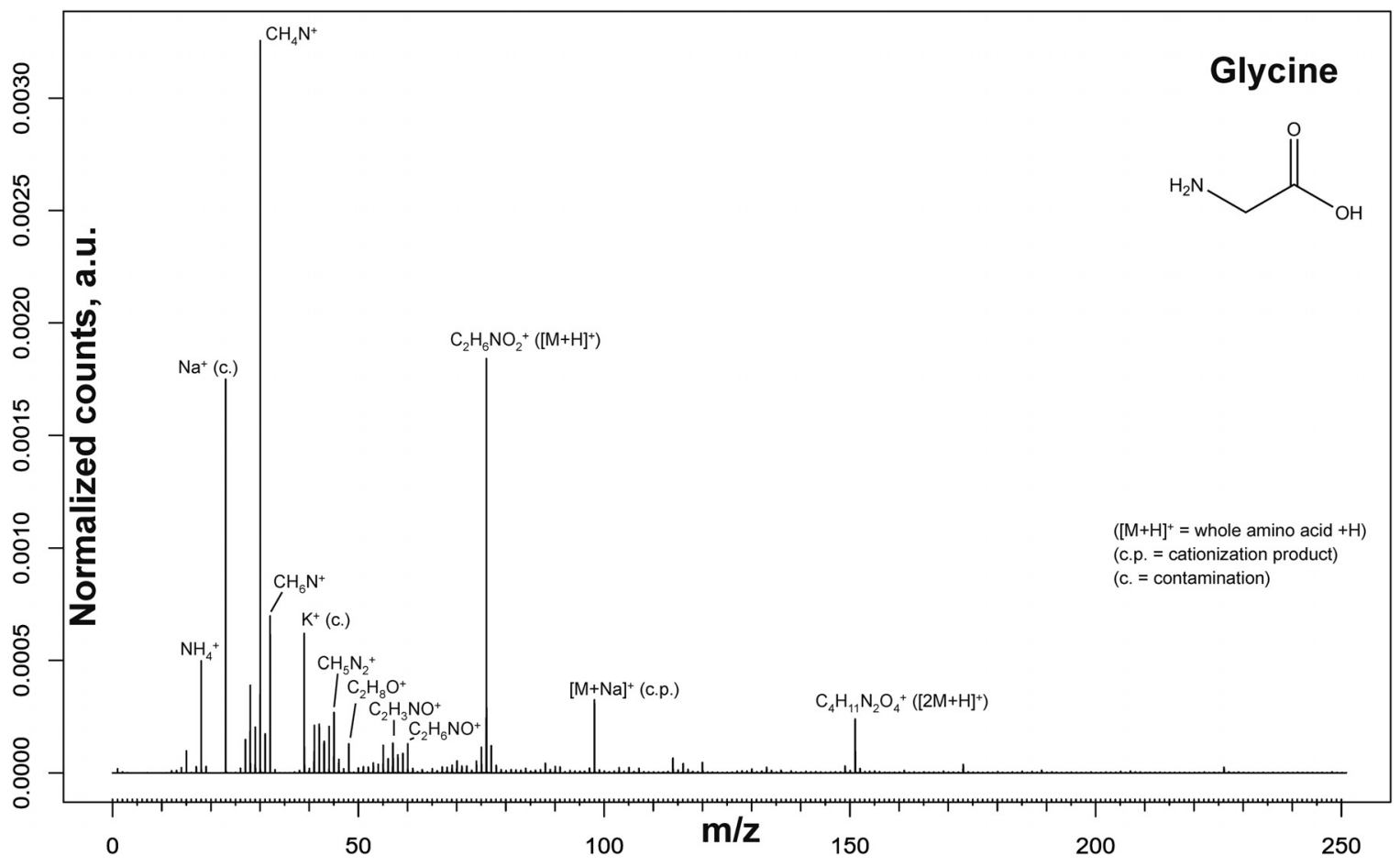

Accession \#

01461-01, 01461-02, 01461-03, 01461-04

Host Material

- Technique

Secondary Source Polarity

Mass Range Minimum

Mass Range Maximum

Species Used for Mass Calibration

Primary lon Dose

Primary Species

Primary lon Pulse Width

Pulsed Beam Current

Beam Raster Size
Silicon wafer

SIMS

Positive

$0 \mathrm{Da}$

$250 \mathrm{Da}$

$\mathrm{CH}_{3}{ }^{+}, \mathrm{NH}_{3}{ }^{+}, \mathrm{NH}_{4}{ }^{+}, \mathrm{CH}_{2} \mathrm{~N}^{+}, \mathrm{C}_{2} \mathrm{H}_{5} \mathrm{NO}_{2}{ }^{+}$

$5.11 \times 10^{11} \mathrm{~cm}^{-2}$

$\mathrm{Bi}_{3}{ }^{+}$

$29.3 \mathrm{~ns}$

$0.0004 \mathrm{nA}$

$200 \times 200 \mu \mathrm{m}^{2}$ 


\begin{tabular}{lccc}
\hline & \multicolumn{2}{c}{ HISTIDINE (HYDROCHLORIDE) } & \\
\hline Spectrum ID \# & Mass (Da) & Species & Peak Assignment \\
\hline $\mathbf{0 1 4 6 2}$ & 81.0447 & $\mathrm{C}_{4} \mathrm{H}_{5} \mathrm{~N}_{2}{ }^{+}$ & Histidine \\
$\ldots$ & 82.0526 & $\mathrm{C}_{4} \mathrm{H}_{6} \mathrm{~N}_{2}{ }^{+}$ & $\ldots$ \\
$\ldots$ & 83.0604 & $\mathrm{C}_{4} \mathrm{H}_{7} \mathrm{~N}_{2}{ }^{+}$ & $\ldots$ \\
$\ldots$ & 93.0447 & $\mathrm{C}_{5} \mathrm{H}_{5} \mathrm{~N}_{2}{ }^{+}$ & $\ldots$ \\
$\ldots$ & 94.0526 & $\mathrm{C}_{5} \mathrm{H}_{6} \mathrm{~N}_{2}{ }^{+}$ & $\ldots$ \\
$\ldots$ & 95.0604 & $\mathrm{C}_{5} \mathrm{H}_{7} \mathrm{~N}_{2}{ }^{+}$ & $\ldots$ \\
$\ldots$ & 110.0713 & $\mathrm{C}_{5} \mathrm{H}_{8} \mathrm{~N}_{3}{ }^{+}$ & $\ldots$ \\
$\ldots$ & 156.0768 & $\mathrm{C}_{6} \mathrm{H}_{10} \mathrm{~N}_{3} \mathrm{O}_{2}{ }^{+}$ & $\ldots$ \\
\hline
\end{tabular}

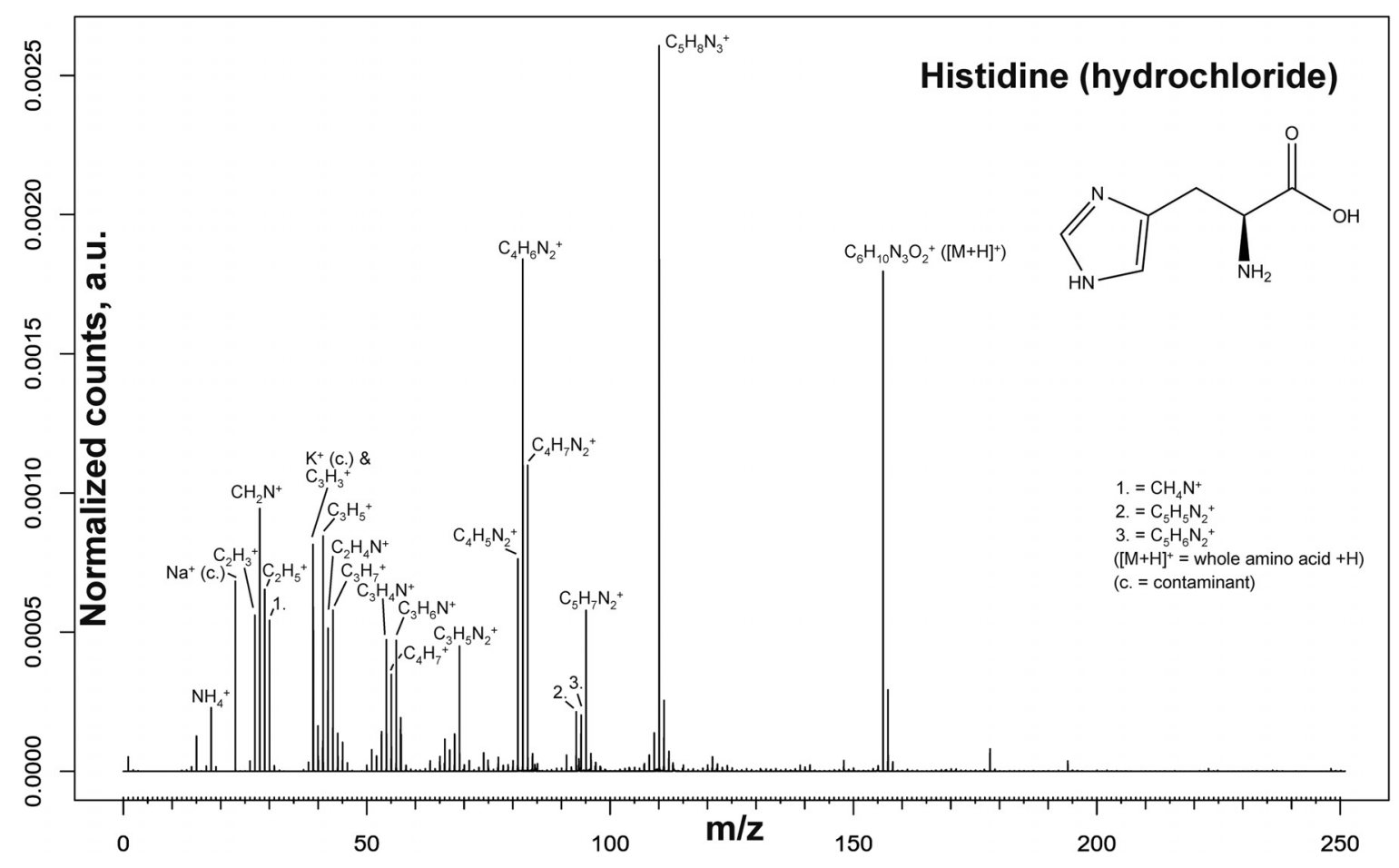

Accession \#

01462-01, 01462-02, 01462-03, 01462-04

Host Material

- Technique

Secondary Source Polarity

Mass Range Minimum

Mass Range Maximum

Species Used for Mass Calibration

Primary lon Dose

Primary Species

Primary lon Pulse Width

Pulsed Beam Current

Beam Raster Size

Silicon wafer
SIMS
Positive
$0 \mathrm{Da}$
$250 \mathrm{Da}$
$\mathrm{CH}_{3}^{+}, \mathrm{NH}_{3}^{+}, \mathrm{NH}_{4}^{+}, \mathrm{CH}_{2} \mathrm{~N}^{+}, \mathrm{C}_{6} \mathrm{H}_{9} \mathrm{~N}_{3} \mathrm{O}_{2}{ }^{+}$
$5.11 \times 10^{11} \mathrm{~cm}^{-2}$
$\mathrm{Bi}_{3}^{+}$
$29.3 \mathrm{~ns}$
$0.0004 \mathrm{nA}$
$200 \times 200 \mu \mathrm{m}^{2}$




\begin{tabular}{lccc}
\hline & \multicolumn{2}{c}{ HYDROXYPROLINE } & \\
\hline Spectrum ID \# & Mass (Da) & Species & Peak Assignment \\
\hline $\mathbf{0 1 4 6 3}$ & 68.0621 & $\mathrm{C}_{5} \mathrm{H}_{8}{ }^{+}$ & Hydroxyproline \\
$\ldots$ & 74.0237 & $\mathrm{C}_{2} \mathrm{H}_{4} \mathrm{NO}_{2}{ }^{+}$ & $\ldots$ \\
$\ldots$ & 84.0444 & $\mathrm{C}_{4} \mathrm{H}_{6} \mathrm{NO}^{+}$ & $\ldots$ \\
$\ldots$ & 86.0600 & $\mathrm{C}_{4} \mathrm{H}_{8} \mathrm{NO}^{+}$ & $\ldots$ \\
$\ldots$ & 87.0679 & $\mathrm{C}_{4} \mathrm{H}_{9} \mathrm{NO}^{+}$ & $\ldots$ \\
$\ldots$ & 88.0757 & $\mathrm{C}_{4} \mathrm{H}_{10} \mathrm{NO}^{+}$ & $\ldots$ \\
$\ldots$ & 114.0550 & $\mathrm{C}_{5} \mathrm{H}_{8} \mathrm{NO}_{2}$ & $\ldots$ \\
$\ldots$ & 116.0706 & $\mathrm{C}_{5} \mathrm{H}_{10} \mathrm{NO}_{2}{ }^{+}$ & $\ldots$ \\
$\ldots$ & 132.0655 & $\mathrm{C}_{5} \mathrm{H}_{10} \mathrm{NO}_{3}{ }^{+}$ & $\ldots$ \\
\hline
\end{tabular}

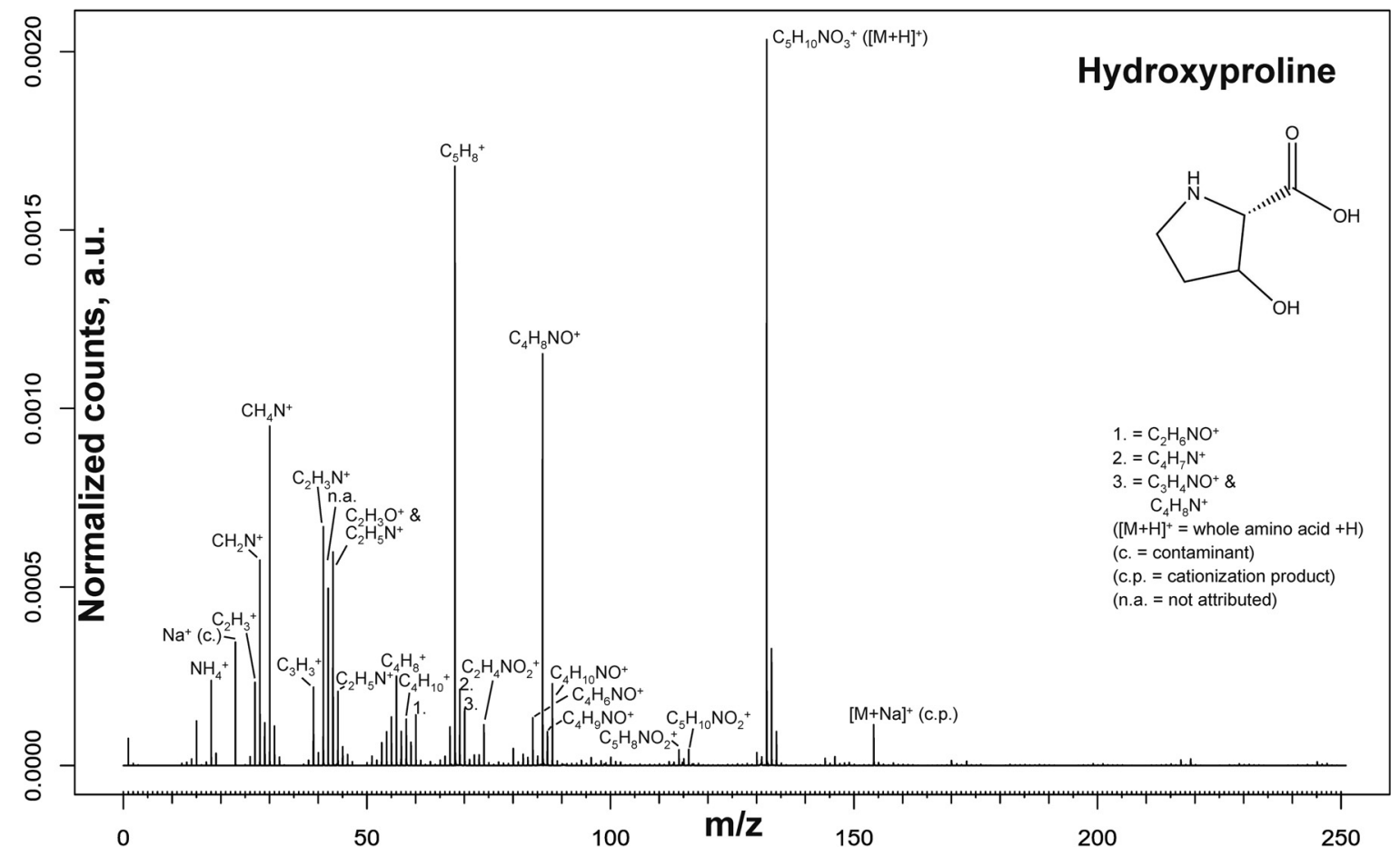

Accession \#

01463-01, 01463-02, 01463-03, 01463-04

Host Material

- Technique

Secondary Source Polarity

Mass Range Minimum

Mass Range Maximum

Species Used for Mass Calibration

Primary lon Dose

Primary Species

Primary lon Pulse Width

Pulsed Beam Current

Beam Raster Size
Silicon wafer

SIMS

Positive

$0 \mathrm{Da}$

$250 \mathrm{Da}$

$\mathrm{CH}_{3}{ }^{+}, \mathrm{NH}_{3}{ }^{+}, \mathrm{NH}_{4}{ }^{+}, \mathrm{C}_{5} \mathrm{H}_{9} \mathrm{NO}_{3}{ }^{+}$

$5.11 \times 10^{11} \mathrm{~cm}^{-2}$

$\mathrm{Bi}_{3}{ }^{+}$

$29.3 \mathrm{~ns}$

$0.0004 \mathrm{nA}$

$200 \times 200 \mu \mathrm{m}^{2}$ 


\begin{tabular}{lccc}
\hline & \multicolumn{3}{c}{ ISOLEUCINE } \\
\hline Spectrum ID \# & Mass (Da) & Species & Peak Assignment \\
\hline $\mathbf{0 1 4 6 4}$ & 69.0699 & $\mathrm{C}_{5} \mathrm{H}_{9}{ }^{+}$ & Isoleucine \\
$\ldots$ & 70.0651 & $\mathrm{C}_{4} \mathrm{H}_{8} \mathrm{~N}^{+}$ & $\ldots$ \\
$\ldots$ & 75.0315 & $\mathrm{C}_{2} \mathrm{H}_{5} \mathrm{NO}_{2}{ }^{+}$ & $\ldots$ \\
$\ldots$ & 84.0808 & $\mathrm{C}_{5} \mathrm{H}_{10} \mathrm{~N}^{+}$ & $\ldots$ \\
$\ldots$ & 86.0964 & $\mathrm{C}_{5} \mathrm{H}_{12} \mathrm{~N}^{+}$ & $\ldots$ \\
$\ldots$ & 87.1043 & $\mathrm{C}_{5} \mathrm{H}_{13} \mathrm{~N}^{+}$ & $\ldots$ \\
$\ldots$ & 132.1019 & $\mathrm{C}_{6} \mathrm{H}_{14} \mathrm{NO}_{2}{ }^{+}$ & $\ldots$ \\
\hline
\end{tabular}

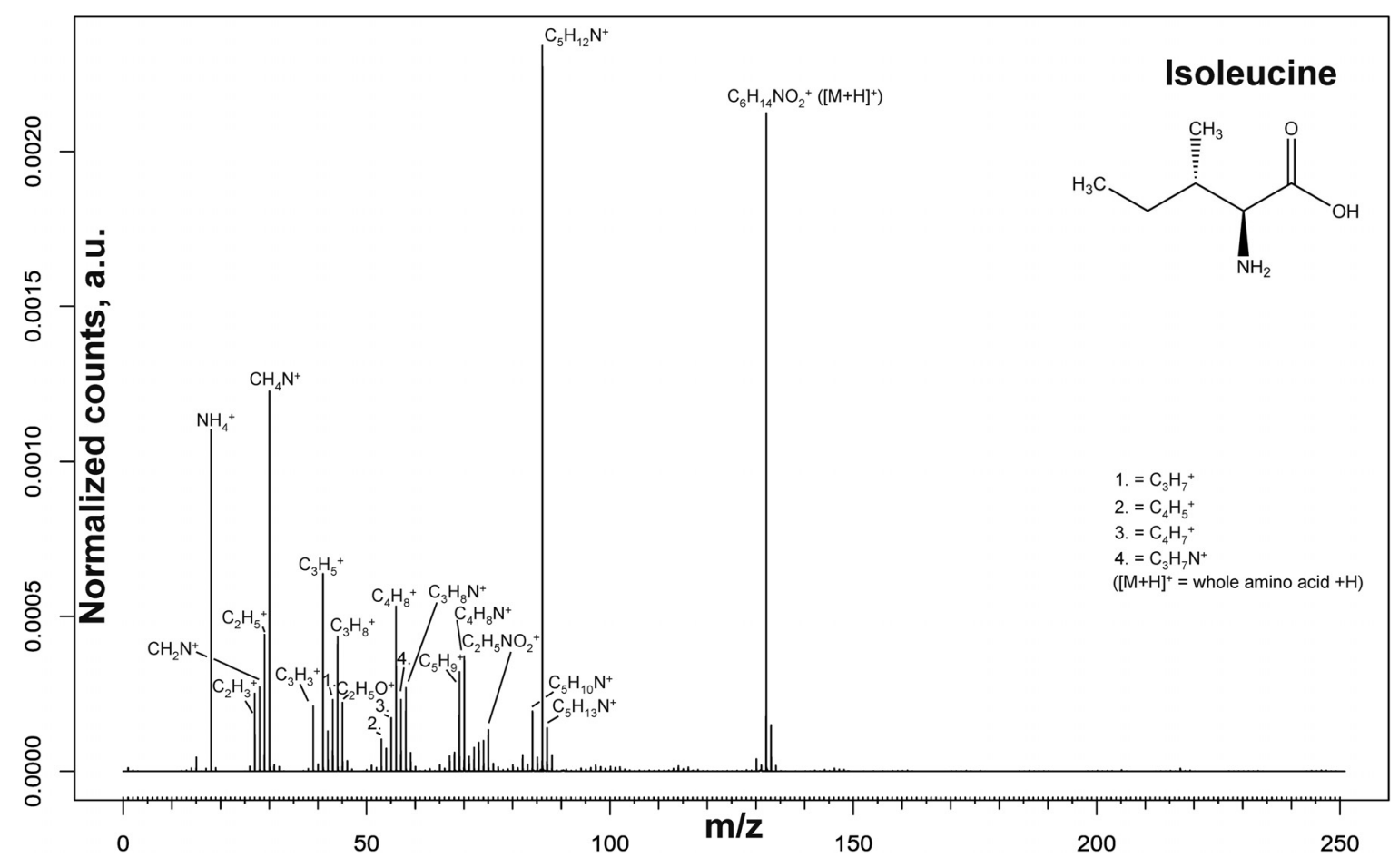

Accession \#

01464-01, 01464-02, 01464-03, 01464-04

Host Material

- Technique

Secondary Source Polarity

Mass Range Minimum

Mass Range Maximum

Species Used for Mass Calibration

Primary lon Dose

Primary Species

Primary lon Pulse Width

Pulsed Beam Current

Beam Raster Size
Silicon wafer

SIMS

Positive

$0 \mathrm{Da}$

$250 \mathrm{Da}$

$\mathrm{CH}_{3}{ }^{+}, \mathrm{NH}_{3}{ }^{+}, \mathrm{CH}_{2} \mathrm{~N}^{+}, \mathrm{C}_{6} \mathrm{H}_{13} \mathrm{NO}_{2}{ }^{+}$

$5.11 \times 10^{11} \mathrm{~cm}^{-2}$

$\mathrm{Bi}_{3}{ }^{+}$

$29.3 \mathrm{~ns}$

$0.0004 \mathrm{nA}$

$200 \times 200 \mu \mathrm{m}^{2}$ 


\begin{tabular}{lccc}
\hline \multicolumn{3}{c}{ LEUCINE } \\
\hline Spectrum ID \# & Mass (Da) & Species & Peak Assignment \\
\hline $\mathbf{0 1 4 6 5}$ & 70.0777 & $\mathrm{C}_{5} \mathrm{H}_{10}{ }^{+}$ & Leucine \\
$\ldots$ & 74.0237 & $\mathrm{C}_{2} \mathrm{H}_{4} \mathrm{NO}_{2}{ }^{+}$ & $\ldots$ \\
$\ldots$ & 84.0808 & $\mathrm{C}_{5} \mathrm{H}_{10} \mathrm{~N}^{+}$ & $\ldots$ \\
$\ldots$ & 86.0964 & $\mathrm{C}_{5} \mathrm{H}_{12} \mathrm{~N}^{+}$ & $\ldots$ \\
$\ldots$ & 132.1019 & $\mathrm{C}_{6} \mathrm{H}_{14} \mathrm{NO}_{2}{ }^{+}$ & $\ldots$ \\
\hline
\end{tabular}

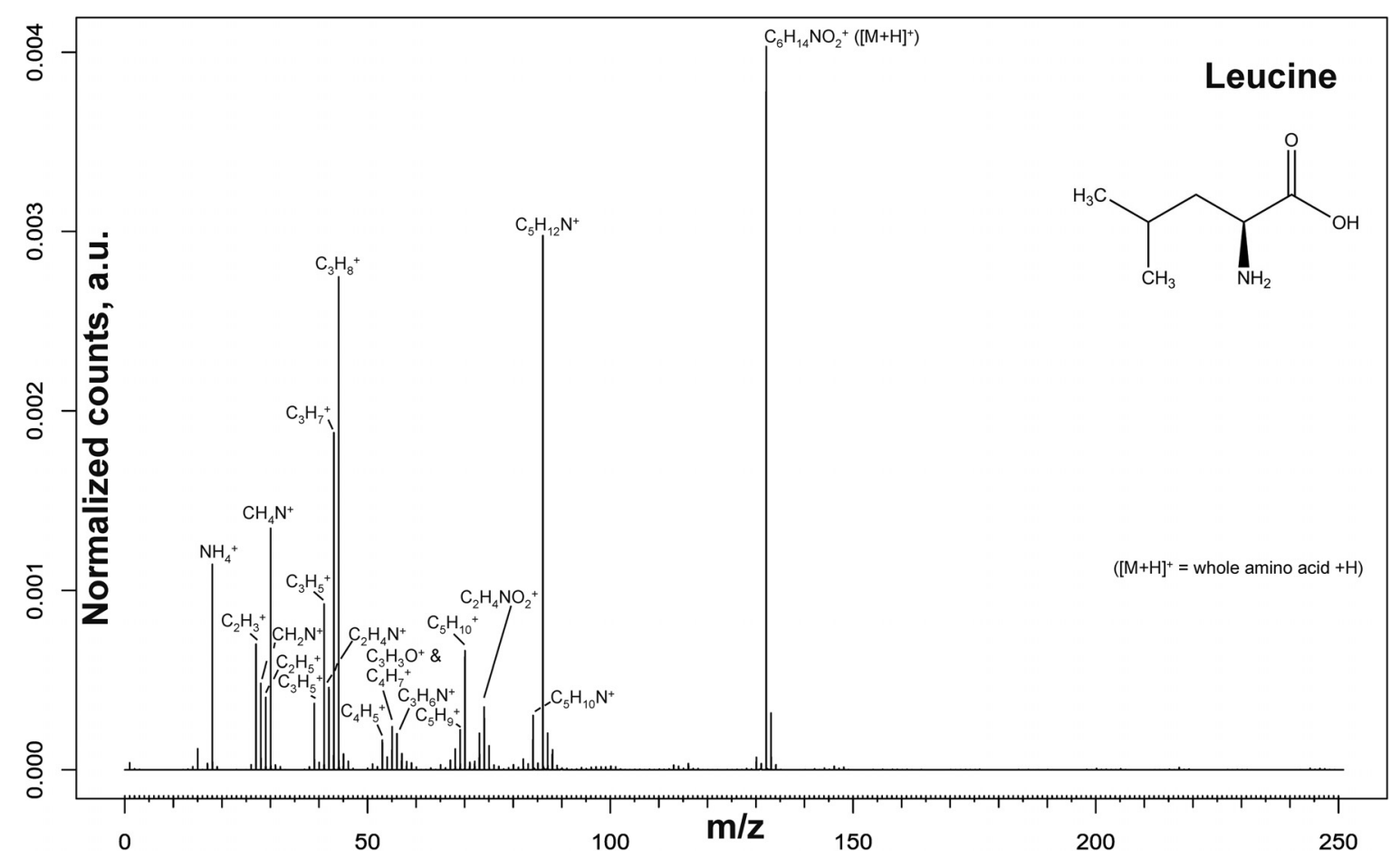

Accession \#

01465-01, 01465-02, 01465-03, 01465-04

Host Material

- Technique

Secondary Source Polarity

Mass Range Minimum

Mass Range Maximum

Species Used for Mass Calibration

Primary lon Dose

Primary Species

Primary lon Pulse Width

Pulsed Beam Current

Beam Raster Size
Silicon wafer

SIMS

Positive

$0 \mathrm{Da}$

$250 \mathrm{Da}$

$\mathrm{CH}_{3}{ }^{+}, \mathrm{NH}_{3}{ }^{+}, \mathrm{CH}_{2} \mathrm{~N}^{+}, \mathrm{C}_{6} \mathrm{H}_{13} \mathrm{NO}_{2}{ }^{+}$

$5.11 \times 10^{11} \mathrm{~cm}^{-2}$

$\mathrm{Bi}_{3}{ }^{+}$

$29.3 \mathrm{~ns}$

$0.0004 \mathrm{nA}$

$200 \times 200 \mu \mathrm{m}^{2}$ 


\section{LYSINE (HYDROCHLORIDE)}

\begin{tabular}{lccc}
\hline Spectrum ID \# & Mass (Da) & Species & Peak Assignment \\
\hline $\mathbf{0 1 4 6 6}$ & 84.0808 & $\mathrm{C}_{5} \mathrm{H}_{10} \mathrm{~N}^{+}$ & Lysine \\
$\ldots$ & 86.0954 & $\mathrm{C}_{5} \mathrm{H}_{12} \mathrm{~N}^{+}$ & $\ldots$ \\
$\ldots$ & 91.0628 & $\mathrm{C}_{3} \mathrm{H}_{9} \mathrm{NO}_{2}{ }^{+}$ & $\ldots$ \\
$\ldots$ & 96.0808 & $\mathrm{C}_{6} \mathrm{H}_{10} \mathrm{~N}^{+}$ & $\ldots$ \\
$\ldots$ & 98.0964 & $\mathrm{C}_{6} \mathrm{H}_{12} \mathrm{~N}^{+}$ & $\ldots$ \\
$\ldots$ & 110.0839 & $\mathrm{C}_{6} \mathrm{H}_{10} \mathrm{~N}_{2}^{+}$ & $\ldots$ \\
$\ldots$ & 128.0706 & $\mathrm{C}_{6} \mathrm{H}_{10} \mathrm{NO}_{2}^{+}$ & $\ldots$ \\
$\ldots$ & 132.1019 & $\mathrm{C}_{6} \mathrm{H}_{14} \mathrm{NO}_{2}^{+}$ & $\ldots$ \\
\hline
\end{tabular}

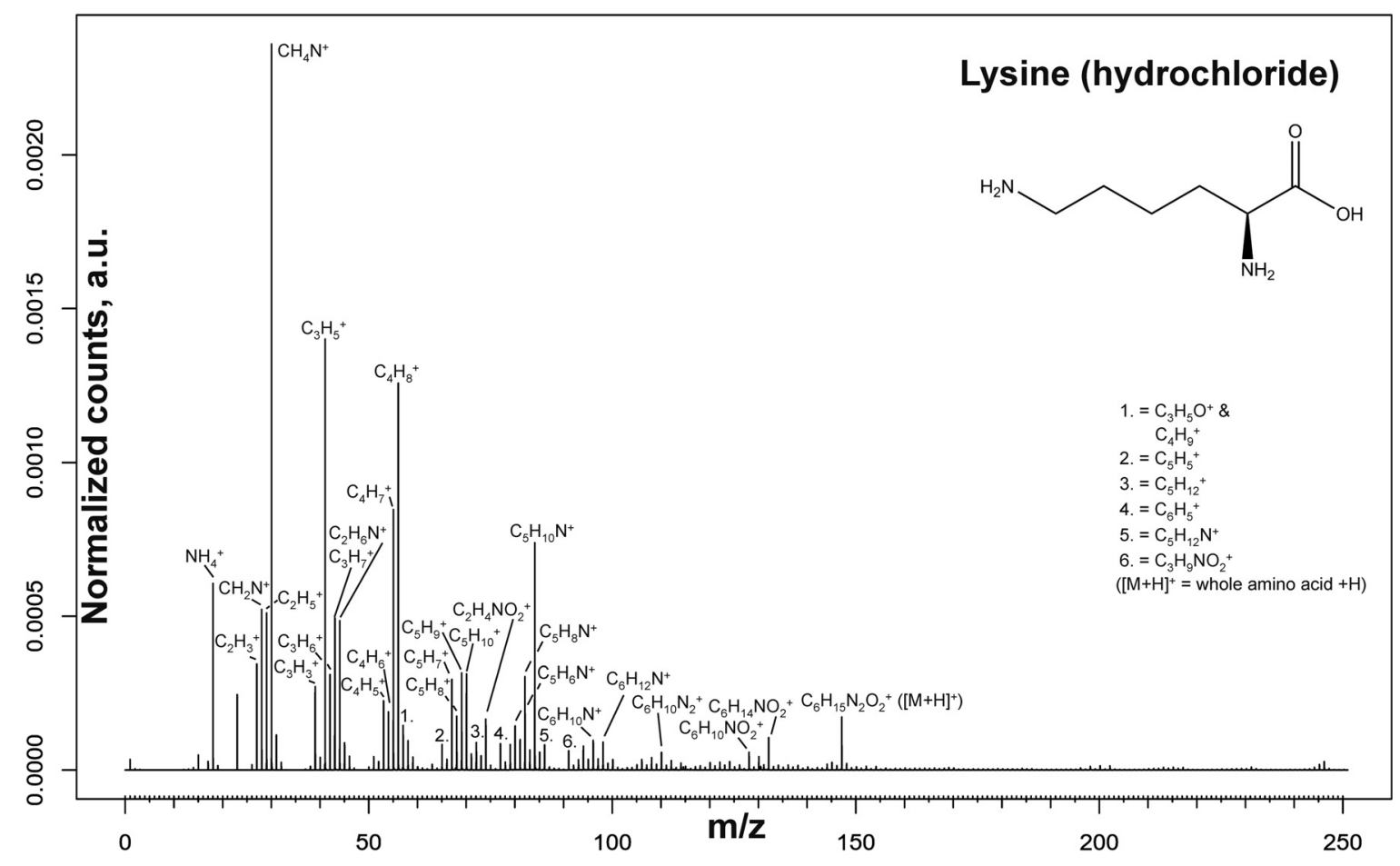

Accession \#

01466-01, 01466-02, 01466-03, 01466-04

Host Material

- Technique

Secondary Source Polarity

Mass Range Minimum

Mass Range Maximum

Species Used for Mass Calibration

Primary lon Dose

Primary Species

Primary lon Pulse Width

Pulsed Beam Current

Beam Raster Size
Silicon wafer

SIMS

Positive

$0 \mathrm{Da}$

$250 \mathrm{Da}$

$\mathrm{CH}_{3}{ }^{+}, \mathrm{NH}_{3}{ }^{+}, \mathrm{NH}_{4}{ }^{+}, \mathrm{CH}_{2} \mathrm{~N}^{+}, \mathrm{C}_{6} \mathrm{H}_{14} \mathrm{~N}_{2} \mathrm{O}_{2}{ }^{+}$

$5.11 \times 10^{11} \mathrm{~cm}^{-2}$

$\mathrm{Bi}_{3}{ }^{+}$

$29.3 \mathrm{~ns}$

$0.0004 \mathrm{nA}$

$200 \times 200 \mu \mathrm{m}^{2}$ 


\begin{tabular}{lccc}
\hline & & METHIONINE & \\
\hline Spectrum ID \# & Mass (Da) & Species & Peak Assignment \\
\hline $\mathbf{0 1 4 6 7}$ & 31.9715 & $\mathrm{~S}^{+}$ & Methionine \\
$\ldots$ & 46.9950 & $\mathrm{CH}_{3} \mathrm{~S}^{+}$ & $\ldots$ \\
$\ldots$ & 61.0106 & $\mathrm{C}_{2} \mathrm{H}_{5} \mathrm{~S}^{+}$ & $\ldots$ \\
$\ldots$ & 74.0185 & $\mathrm{C}_{3} \mathrm{H}_{6} \mathrm{~S}^{+}$ & $\ldots$ \\
$\ldots$ & 75.0315 & $\mathrm{C}_{2} \mathrm{H}_{5} \mathrm{NO}_{2}$ & $\ldots$ \\
$\ldots$ & 88.0215 & $\mathrm{C}_{3} \mathrm{H}_{6} \mathrm{NS}^{+}$ & $\ldots$ \\
$\ldots$ & 88.0341 & $\mathrm{C}_{4} \mathrm{H}_{8} \mathrm{~S}^{+}$ & $\ldots$ \\
$\ldots$ & 102.0550 & $\mathrm{C}_{4} \mathrm{H}_{8} \mathrm{NO}_{2}$ & $\ldots$ \\
$\ldots$ & 104.0528 & $\mathrm{C}_{4} \mathrm{H}_{10} \mathrm{NS}^{+}$ & $\ldots$ \\
$\ldots$ & 133.0318 & $\mathrm{C}_{5} \mathrm{H}_{9} \mathrm{O}_{2} \mathrm{~S}^{+}$ & $\ldots$ \\
\hline
\end{tabular}

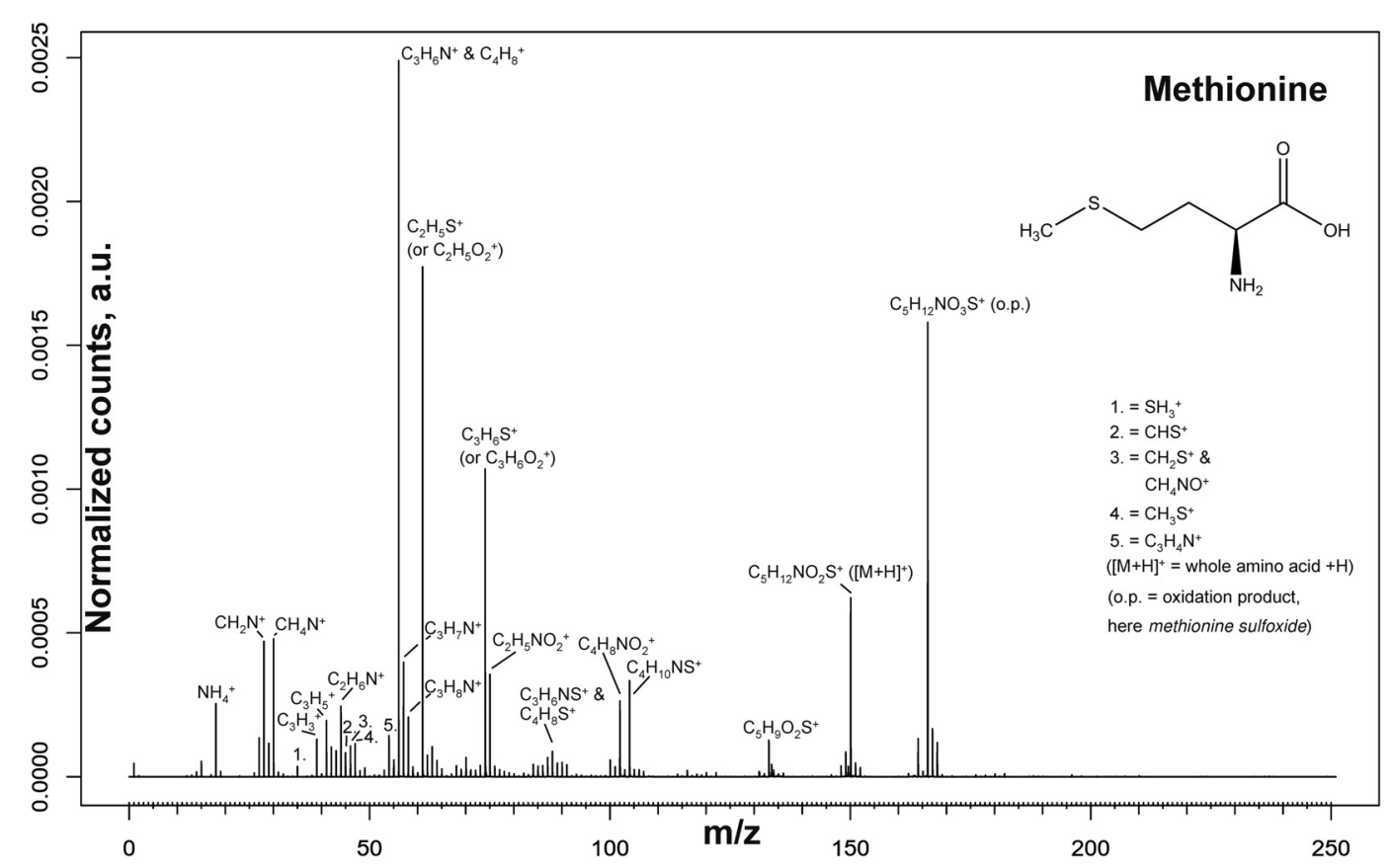

\begin{tabular}{|c|c|}
\hline Accession \# & $01467-01,01467-02,01467-03,01467-04$ \\
\hline Host Material & Silicon wafer \\
\hline Technique & SIMS \\
\hline Secondary Source Polarity & Positive \\
\hline Mass Range Minimum & $0 \mathrm{Da}$ \\
\hline Mass Range Maximum & $250 \mathrm{Da}$ \\
\hline Species Used for Mass Calibration & $\mathrm{CH}_{3}{ }^{+}, \mathrm{NH}_{3}{ }^{+}, \mathrm{NH}_{4}^{+}, \mathrm{CH}_{2} \mathrm{~N}^{+}, \mathrm{C}_{5} \mathrm{H}_{13} \mathrm{SNO}_{2}{ }^{+}$ \\
\hline Primary Ion Dose & $5.11 \times 10^{11} \mathrm{~cm}^{-2}$ \\
\hline Primary Species & $\mathrm{Bi}_{3}^{+}$ \\
\hline Primary Ion Pulse Width & $29.3 \mathrm{~ns}$ \\
\hline Pulsed Beam Current & $0.0004 \mathrm{nA}$ \\
\hline Beam Raster Size & $200 \times 200 \mu \mathrm{m}^{2}$ \\
\hline Comment & $\begin{array}{l}\text { The assignments } \mathrm{C}_{2} \mathrm{H}_{5} \mathrm{O}_{2}{ }^{+} \text {and } \mathrm{C}_{3} \mathrm{H}_{6} \mathrm{O}_{2+} \text { (in brackets) show a substantially } \\
\text { lower deviation but are, in the author's judgment, sufficiently inconsistent with } \\
\text { the fragmentation patterns observed in other amino } \\
\text { acids to warrant the choice of peak attribution made above. }\end{array}$ \\
\hline
\end{tabular}




\begin{tabular}{lccc}
\hline & \multicolumn{2}{c}{ PHENYLALANINE } \\
\hline Spectrum ID \# & Mass (Da) & Species & Peak Assignment \\
\hline $\mathbf{0 1 4 6 8}$ & 77.0386 & $\mathrm{C}_{6} \mathrm{H}_{5}{ }^{+}$ & Phenylalanine \\
$\ldots$ & 91.0628 & $\mathrm{C}_{3} \mathrm{H}_{9} \mathrm{NO}_{2}^{+}$ & $\ldots$ \\
$\ldots$ & 103.0542 & $\mathrm{C}_{8} \mathrm{H}_{7}^{+}$ & $\ldots$ \\
$\ldots$ & 105.0699 & $\mathrm{C}_{8} \mathrm{H}_{9}^{+}$ & $\ldots$ \\
$\ldots$ & 107.0491 & $\mathrm{C}_{7} \mathrm{H}_{7} \mathrm{O}^{+}$ & $\ldots$ \\
$\ldots$ & 115.0628 & $\mathrm{C}_{5} \mathrm{H}_{9} \mathrm{NO}_{2}^{+}$ & $\ldots$ \\
$\ldots$ & 120.0808 & $\mathrm{C}_{8} \mathrm{H}_{10} \mathrm{~N}^{+}$ & $\ldots$ \\
$\ldots$ & 121.0886 & $\mathrm{C}_{8} \mathrm{H}_{11} \mathrm{~N}^{+}$ & $\ldots$ \\
$\ldots$ & 166.0863 & $\mathrm{C}_{9} \mathrm{H}_{12} \mathrm{NO}_{2}{ }^{+}$ & $\ldots$ \\
\hline
\end{tabular}

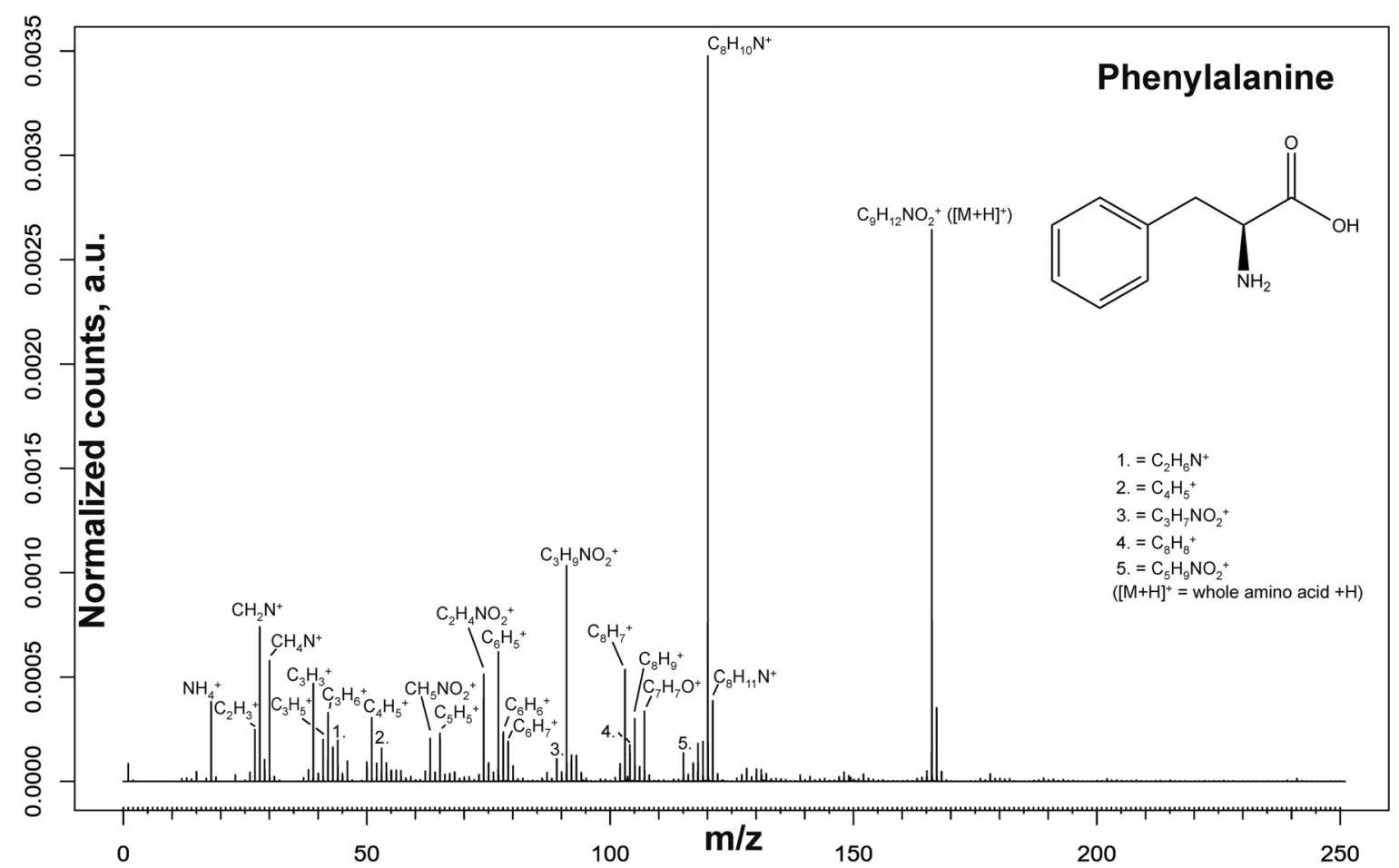

\begin{tabular}{rc}
\hline Accession \# & $\mathbf{0 1 4 6 8 - 0 1 , 0 1 4 6 8 - 0 2 , 0 1 4 6 8 - 0 3 , 0 1 4 6 8 - 0 4}$ \\
\hline Host Material & Silicon wafer \\
$\mathbf{\text { Technique }}$ & $\mathrm{SIMS}$ \\
Secondary Source Polarity & Positive \\
Mass Range Minimum & $0 \mathrm{Da}$ \\
Mass Range Maximum & $250 \mathrm{Da}$ \\
Species Used for Mass Calibration & $\mathrm{CH}_{3}{ }^{+}, \mathrm{NH}_{3}{ }^{+}, \mathrm{NH}_{4}{ }^{+}, \mathrm{CH}_{2} \mathrm{~N}^{+}, \mathrm{C}_{9} \mathrm{H}_{11} \mathrm{NO}_{2}{ }^{+}$ \\
Primary lon Dose & $5.11 \times 10^{11} \mathrm{~cm}^{-2}$ \\
Primary Species & $\mathrm{Bi}_{3}{ }^{+}$ \\
Primary lon Pulse Width & $29.3 \mathrm{~ns}$ \\
Pulsed Beam Current & $0.0004 \mathrm{nA}^{2}$ \\
Beam Raster Size & $200 \times 200 \mu \mathrm{m}^{2}$ \\
\hline
\end{tabular}




\begin{tabular}{lccc}
\hline & \multicolumn{3}{c}{ PROLINE } \\
\hline Spectrum ID \# & Mass (Da) & Species & Peak Assignment \\
\hline $\mathbf{0 1 4 6 9}$ & 68.0621 & $\mathrm{C}_{5} \mathrm{H}_{8}{ }^{+}$ & Proline \\
$\ldots$ & 70.0651 & $\mathrm{C}_{4} \mathrm{H}_{8} \mathrm{~N}^{+}$ & $\ldots$ \\
$\ldots$ & 70.0777 & $\mathrm{C}_{5} \mathrm{H}_{10}{ }^{+}$ & $\ldots$ \\
$\ldots$ & 71.0730 & $\mathrm{C}_{4} \mathrm{H}_{9} \mathrm{~N}^{+}$ & $\ldots$ \\
$\ldots$ & 72.0808 & $\mathrm{C}_{4} \mathrm{H}_{10} \mathrm{~N}^{+}$ & $\ldots$ \\
$\ldots$ & 116.0706 & $\mathrm{C}_{5} \mathrm{H}_{10} \mathrm{NO}_{2}{ }^{+}$ & $\ldots$ \\
\hline
\end{tabular}

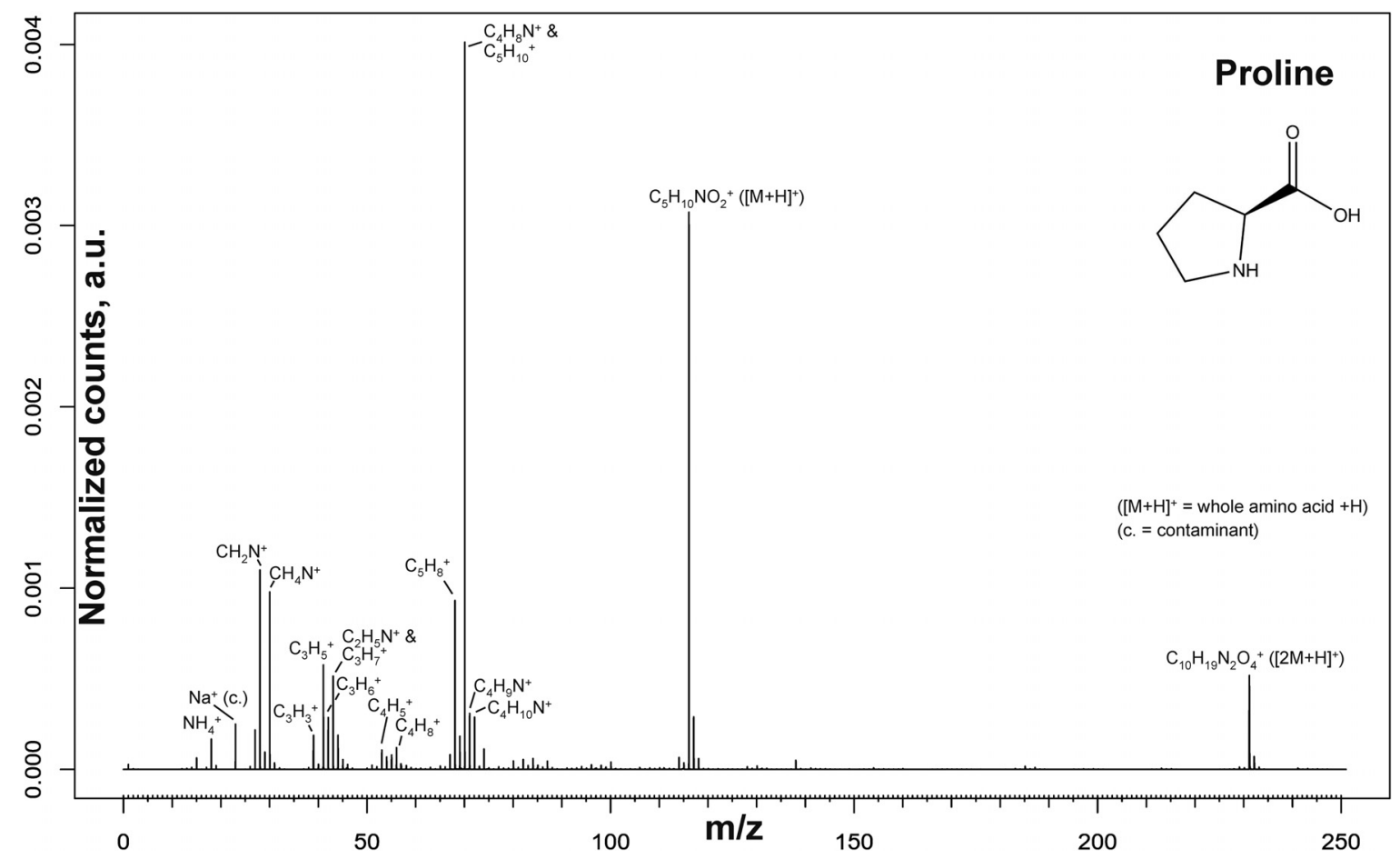

Accession \#

01469-01, 01469-02, 01469-03, 01469-04

Host Material

- Technique

Secondary Source Polarity

Mass Range Minimum

Mass Range Maximum

Species Used for Mass Calibration

Primary lon Dose

Primary Species

Primary lon Pulse Width

Pulsed Beam Current

Beam Raster Size
Silicon wafer

SIMS

Positive

$0 \mathrm{Da}$

$250 \mathrm{Da}$

$\mathrm{CH}_{3}{ }^{+}, \mathrm{NH}_{3}{ }^{+}, \mathrm{NH}_{4}^{+}, \mathrm{C}_{5} \mathrm{H}_{9} \mathrm{NO}_{2}{ }^{+}$

$5.11 \times 10^{11} \mathrm{~cm}^{-2}$

$\mathrm{Bi}_{3}{ }^{+}$

$29.3 \mathrm{~ns}$

$0.0004 \mathrm{nA}$

$200 \times 200 \mu \mathrm{m}^{2}$ 


\begin{tabular}{lccc}
\hline \multicolumn{3}{c}{ SERINE } \\
\hline Spectrum ID \# & Mass (Da) & Species & Peak Assignment \\
\hline $\mathbf{0 1 4 7 0}$ & 60.0444 & $\mathrm{C}_{2} \mathrm{H}_{6} \mathrm{NO}^{+}$ & Serine \\
$\ldots$ & 70.0287 & $\mathrm{C}_{3} \mathrm{H}_{4} \mathrm{NO}^{+}$ & $\ldots$ \\
$\ldots$ & 73.0284 & $\mathrm{C}_{3} \mathrm{H}_{5} \mathrm{O}_{2}^{+}$ & $\ldots$ \\
$\ldots$ & 88.0393 & $\mathrm{C}_{3} \mathrm{H}_{6} \mathrm{NO}_{2}^{+}$ & $\ldots$ \\
$\ldots$ & 106.0499 & $\mathrm{C}_{3} \mathrm{H}_{8} \mathrm{NO}_{3}^{+}$ & $\ldots$ \\
\hline
\end{tabular}

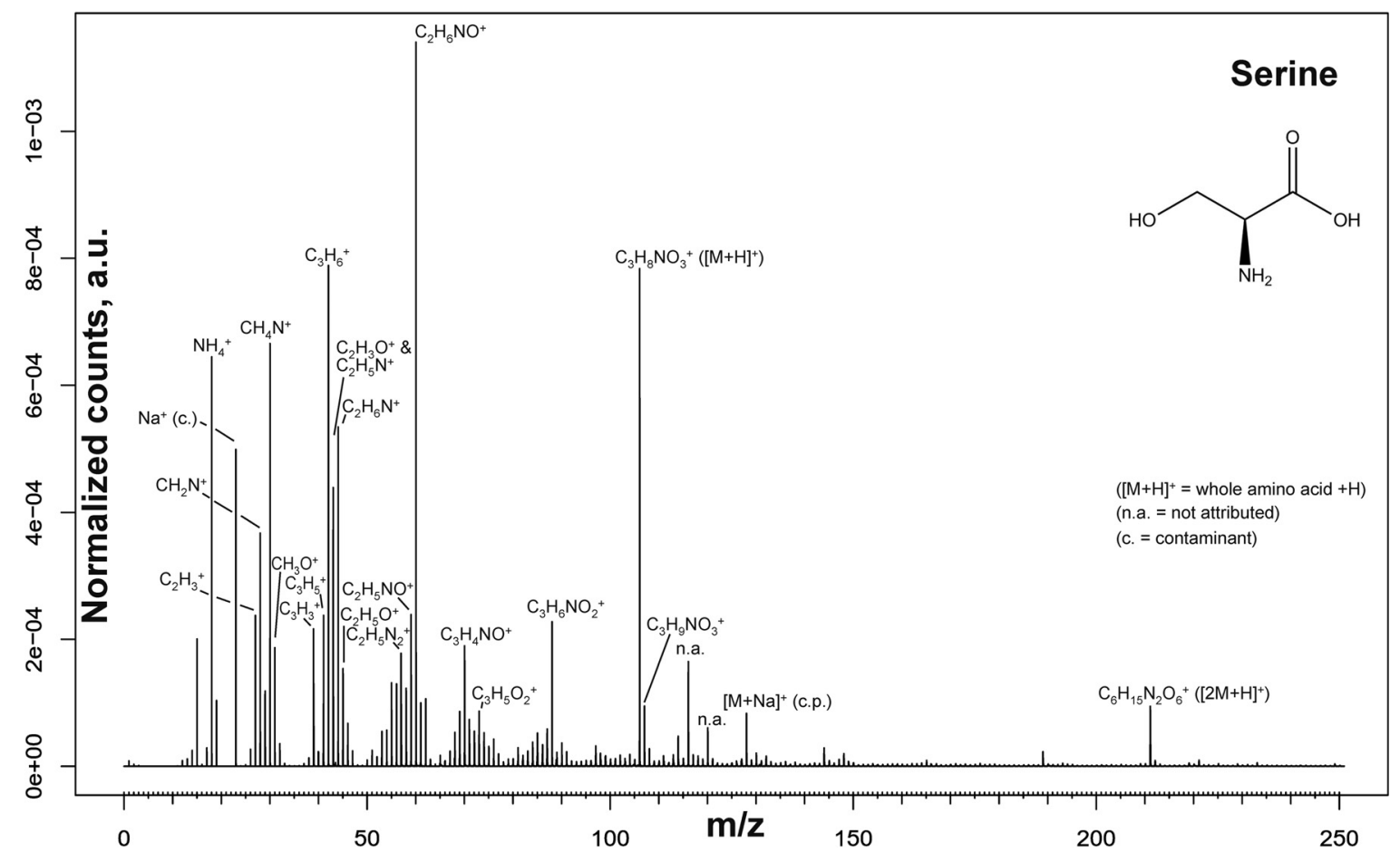

Accession \#

Host Material

Technique

Secondary Source Polarity

Mass Range Minimum

Mass Range Maximum

Species Used for Mass Calibration

Primary Ion Dose

Primary Species

Primary Ion Pulse Width

Pulsed Beam Current

Beam Raster Size
01470-01, 01470-02, 01470-03, 01470-04

Silicon wafer

SIMS

Positive

$0 \mathrm{Da}$

$250 \mathrm{Da}$

$\mathrm{CH}_{3}{ }^{+}, \mathrm{NH}_{3}{ }^{+}, \mathrm{NH}_{4}{ }^{+}, \mathrm{CH}_{2} \mathrm{~N}^{+}, \mathrm{C}_{3} \mathrm{H}_{9} \mathrm{NO}_{3}{ }^{+}$

$5.11 \times 10^{11} \mathrm{~cm}^{-2}$

$\mathrm{Bi}_{3}{ }^{+}$

29.3 ns

$0.0004 \mathrm{nA}$

$200 \times 200 \mu \mathrm{m}^{2}$ 


\begin{tabular}{lccc}
\hline \multicolumn{3}{c}{ THREONINE } \\
\hline Spectrum ID \# & Mass (Da) & Species & Peak Assignment \\
\hline $\mathbf{0 1 4 7 1}$ & 56.0621 & $\mathrm{C}_{4} \mathrm{H}_{8}{ }^{+}$ & Threonine \\
$\ldots$ & 74.0600 & $\mathrm{C}_{3} \mathrm{H}_{8} \mathrm{NO}^{+}$ & $\ldots$ \\
$\ldots$ & 84.0444 & $\mathrm{C}_{4} \mathrm{H}_{6} \mathrm{NO}^{+}$ & $\ldots$ \\
$\ldots$ & 102.0550 & $\mathrm{C}_{4} \mathrm{H}_{8} \mathrm{NO}_{2}{ }^{+}$ & $\ldots$ \\
$\ldots$ & 120.0655 & $\mathrm{C}_{4} \mathrm{H}_{10} \mathrm{NO}_{3}{ }^{+}$ & $\ldots$ \\
\hline
\end{tabular}

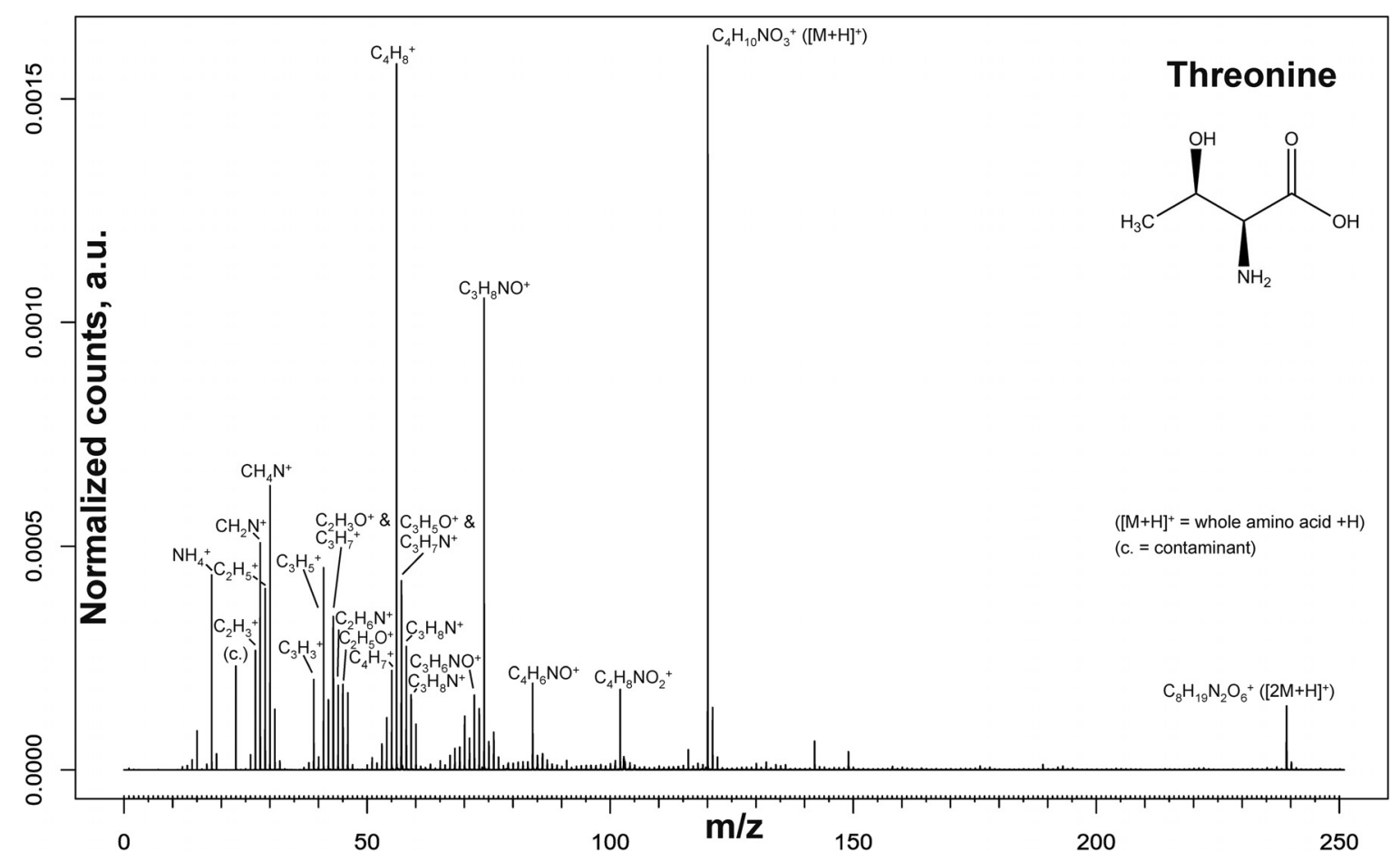

\begin{tabular}{rc}
\hline Accession \# & 01471-01, 01471-02, 01471-03, 01471-04 \\
\hline Host Material & Silicon wafer \\
Technique & SIMS \\
Secondary Source Polarity & Positive \\
Mass Range Minimum & $0 \mathrm{Da}$ \\
Mass Range Maximum & $250 \mathrm{Da}$ \\
Species Used for Mass Calibration & $\mathrm{CH}_{3}^{+}, \mathrm{NH}_{3}{ }^{+}, \mathrm{NH}_{4}{ }^{+}, \mathrm{CH}_{2} \mathrm{~N}^{+}, \mathrm{C}_{4} \mathrm{H}_{11} \mathrm{NO}_{3}{ }^{+}$ \\
Primary lon Dose & $5.11 \times 10^{11} \mathrm{~cm}^{-2}$ \\
Primary Species & $\mathrm{Bi}_{3}^{+}$ \\
Primary lon Pulse Width & $29.3 \mathrm{~ns}^{+}$ \\
Pulsed Beam Current & $0.0004 \mathrm{nA}^{2}$ \\
Beam Raster Size & $200 \times 200 \mu \mathrm{m}^{2}$ \\
\hline
\end{tabular}




\begin{tabular}{|c|c|c|c|}
\hline \multicolumn{4}{|c|}{ TRYPTOPHAN } \\
\hline Spectrum ID \# & Mass (Da) & Species & Peak Assignment \\
\hline 01472 & 77.0386 & $\mathrm{C}_{6} \mathrm{H}_{5}^{+}$ & Tryptophan \\
\hline$\ldots$ & 115.0417 & $\mathrm{C}_{8} \mathrm{H}_{5} \mathrm{~N}^{+}$ & $\ldots$ \\
\hline$\ldots$ & 117.0573 & $\mathrm{C}_{8} \mathrm{H}_{7} \mathrm{~N}^{+}$ & $\ldots$ \\
\hline$\ldots$ & 118.0651 & $\mathrm{C}_{8} \mathrm{H}_{8} \mathrm{~N}^{+}$ & $\ldots$ \\
\hline$\ldots$ & 130.0651 & $\mathrm{C}_{9} \mathrm{H}_{8} \mathrm{~N}^{+}$ & $\ldots$ \\
\hline$\ldots$ & 132.0808 & $\mathrm{C}_{9} \mathrm{H}_{10} \mathrm{~N}^{+}$ & $\ldots$ \\
\hline$\ldots$ & 143.0730 & $\mathrm{C}_{10} \mathrm{H}_{9} \mathrm{~N}^{+}$ & $\ldots$ \\
\hline$\ldots$ & 144.0682 & $\mathrm{C}_{9} \mathrm{H}_{8} \mathrm{~N}_{2}^{+}$ & $\ldots$ \\
\hline$\ldots$ & 169.0917 & $\mathrm{C}_{10} \mathrm{H}_{11} \mathrm{~N}_{2}{ }^{+}$ & $\ldots$ \\
\hline$\ldots$ & 188.0944 & $\mathrm{C}_{11} \mathrm{H}_{12} \mathrm{~N}_{2} \mathrm{O}^{+}$ & $\ldots$ \\
\hline$\ldots$ & 205.0972 & $\mathrm{C}_{11} \mathrm{H}_{13} \mathrm{~N}_{2} \mathrm{O}_{2}^{+}$ & $\ldots$ \\
\hline
\end{tabular}

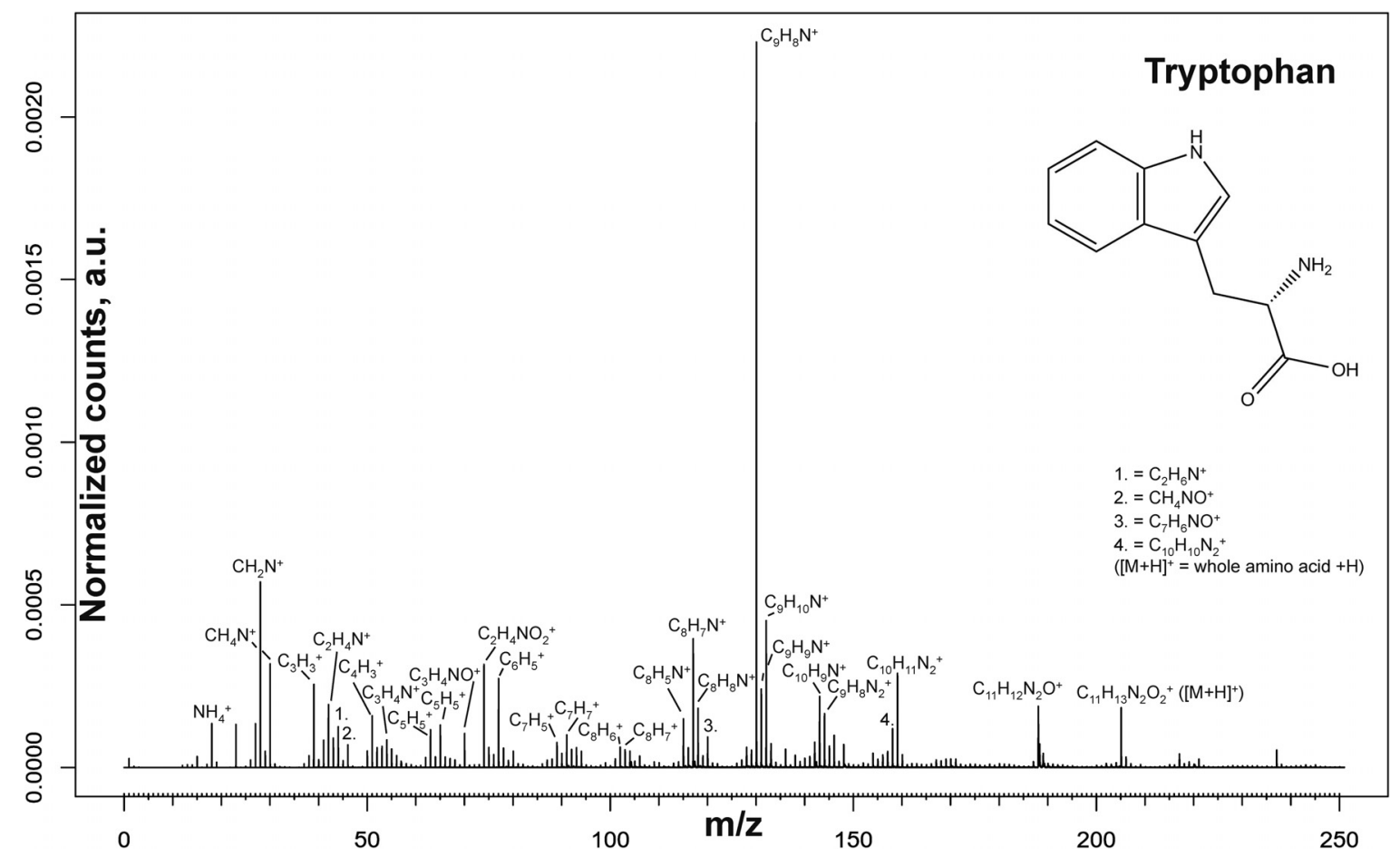

\begin{tabular}{rc}
\hline Accession \# & 01472-01, 01472-02, 01472-03, 01472-04 \\
\hline Host Material & Silicon wafer \\
Technique & SIMS \\
Secondary Source Polarity & Positive \\
Mass Range Minimum & $0 \mathrm{Da}$ \\
Mass Range Maximum & $250 \mathrm{Da}$ \\
Species Used for Mass Calibration & $\mathrm{CH}_{3}{ }^{+}, \mathrm{NH}_{3}{ }^{+}, \mathrm{NH}_{4}^{+}, \mathrm{CH}_{2} \mathrm{~N}^{+}, \mathrm{C}_{11} \mathrm{H}_{13} \mathrm{~N}_{2} \mathrm{O}_{2}{ }^{+}$ \\
Primary lon Dose & $4.73 \times 10^{11} \mathrm{~cm}^{-2}$ \\
Primary Species & $\mathrm{Bi}_{3}{ }^{+}$ \\
Primary lon Pulse Width & $29.3 \mathrm{~ns}^{-}$ \\
Pulsed Beam Current & $0.00037 \mathrm{nA}^{2}$ \\
Beam Raster Size & $200 \times 200 \mu \mathrm{m}^{2}$ \\
\hline
\end{tabular}




\begin{tabular}{|c|c|c|c|}
\hline \multicolumn{4}{|c|}{ TYROSINE } \\
\hline Spectrum ID \# & Mass (Da) & Species & Peak Assignment \\
\hline 01473 & 77.0386 & $\mathrm{C}_{6} \mathrm{H}_{5}^{+}$ & Tyrosine \\
\hline$\ldots$ & 91.0542 & $\mathrm{C}_{7} \mathrm{H}_{7}^{+}$ & $\ldots$ \\
\hline$\ldots$ & 107.0491 & $\mathrm{C}_{7} \mathrm{H}_{7} \mathrm{O}^{+}$ & $\ldots$ \\
\hline$\ldots$ & 115.0417 & $\mathrm{C}_{8} \mathrm{H}_{5} \mathrm{~N}^{+}$ & $\ldots$ \\
\hline$\ldots$ & 121.0648 & $\mathrm{C}_{8} \mathrm{H}_{9} \mathrm{O}^{+}$ & $\ldots$ \\
\hline$\ldots$ & 136.0757 & $\mathrm{C}_{8} \mathrm{H}_{10} \mathrm{NO}^{+}$ & $\ldots$ \\
\hline$\ldots$ & 149.0107 & $\mathrm{C}_{7} \mathrm{H}_{3} \mathrm{NO}_{3}{ }^{+}$ & $\ldots$ \\
\hline$\ldots$ & 165.0546 & $\mathrm{C}_{9} \mathrm{H}_{9} \mathrm{O}_{3}{ }^{+}$ & $\ldots$ \\
\hline$\ldots$ & 182.0812 & $\mathrm{C}_{9} \mathrm{H}_{12} \mathrm{NO}_{3}{ }^{+}$ & $\ldots$ \\
\hline
\end{tabular}

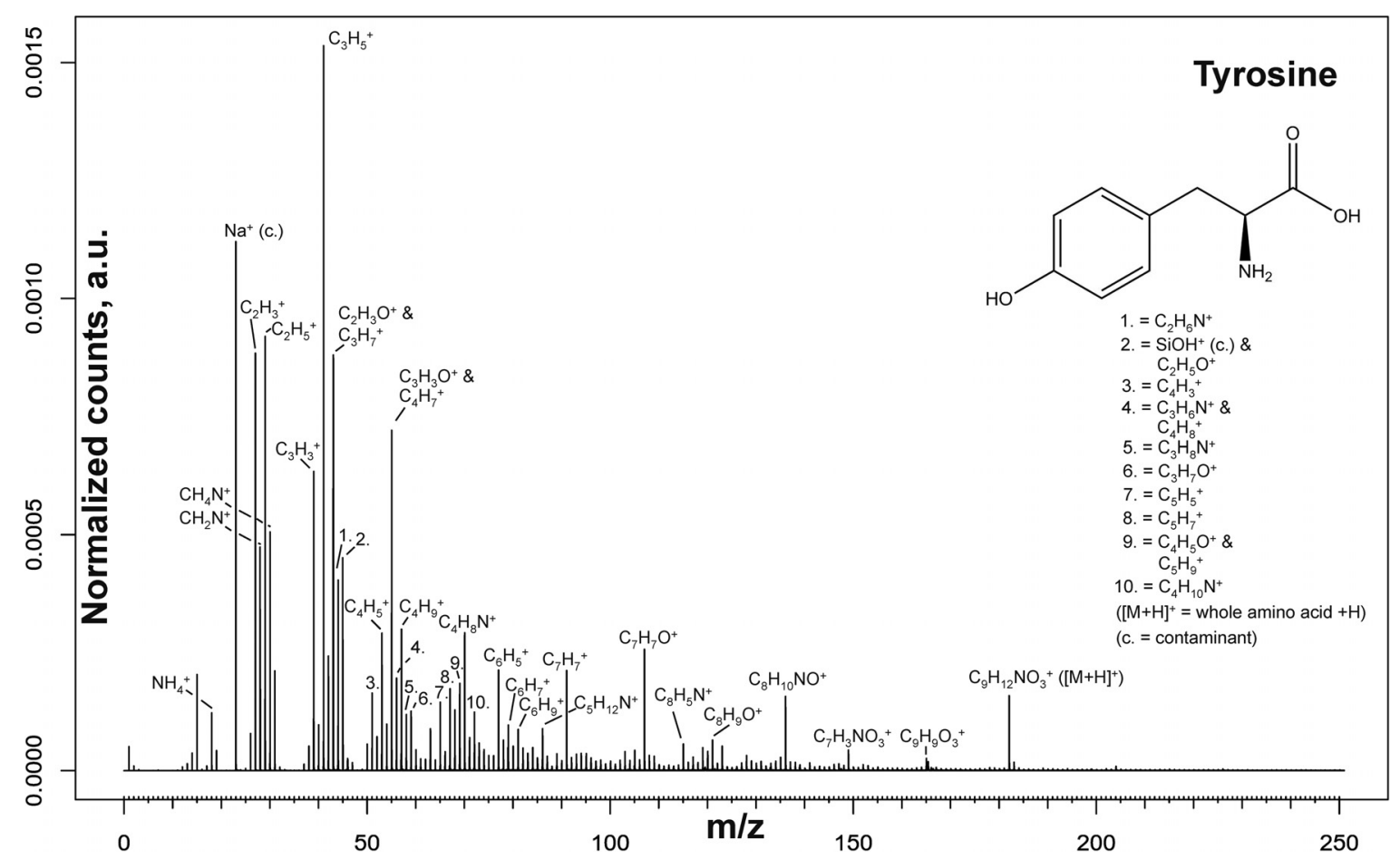

\begin{tabular}{|c|c|}
\hline Accession \# & 01473-01, 01473-02, 01473-03, 01473-04 \\
\hline E Host Material & Silicon wafer \\
\hline Technique & SIMS \\
\hline Secondary Source Polarity & Positive \\
\hline Mass Range Minimum & $0 \mathrm{Da}$ \\
\hline Mass Range Maximum & $250 \mathrm{Da}$ \\
\hline Species Used for Mass Calibration & $\mathrm{CH}_{3}{ }^{+}, \mathrm{NH}_{3}{ }^{+}, \mathrm{NH}_{4}{ }^{+}, \mathrm{CH}_{2} \mathrm{~N}^{+}, \mathrm{C}_{9} \mathrm{H}_{12} \mathrm{NO}_{3}{ }^{+}$ \\
\hline Primary lon Dose & $4.73 \times 10^{11} \mathrm{~cm}^{-2}$ \\
\hline Primary Species & $\mathrm{Bi}_{3}^{+}$ \\
\hline Primary Ion Pulse Width & $29.3 \mathrm{~ns}$ \\
\hline Pulsed Beam Current & $0.00037 \mathrm{nA}$ \\
\hline Beam Raster Size & $200 \times 200 \mu \mathrm{m}^{2}$ \\
\hline
\end{tabular}




\begin{tabular}{lccc}
\hline & \multicolumn{3}{c}{ VALINE } \\
\hline Spectrum ID \# & Mass (Da) & Species & Peak Assignment \\
\hline $\mathbf{0 1 4 7 4}$ & 55.0542 & $\mathrm{C}_{4} \mathrm{H}_{7}{ }^{+}$ & Valine \\
$\ldots$ & 68.0495 & $\mathrm{C}_{4} \mathrm{H}_{6} \mathrm{~N}^{+}$ & $\ldots$ \\
$\ldots$ & 72.0808 & $\mathrm{C}_{4} \mathrm{H}_{10} \mathrm{~N}^{+}$ & $\ldots$ \\
$\ldots$ & 73.0886 & $\mathrm{C}_{4} \mathrm{H}_{11} \mathrm{~N}^{+}$ & $\ldots$ \\
$\ldots$ & 75.0315 & $\mathrm{C}_{2} \mathrm{H}_{5} \mathrm{NO}_{2}^{+}$ & $\ldots$ \\
$\ldots$ & 118.0862 & $\mathrm{C}_{5} \mathrm{H}_{12} \mathrm{NO}_{2}{ }^{+}$ & $\ldots$ \\
\hline
\end{tabular}

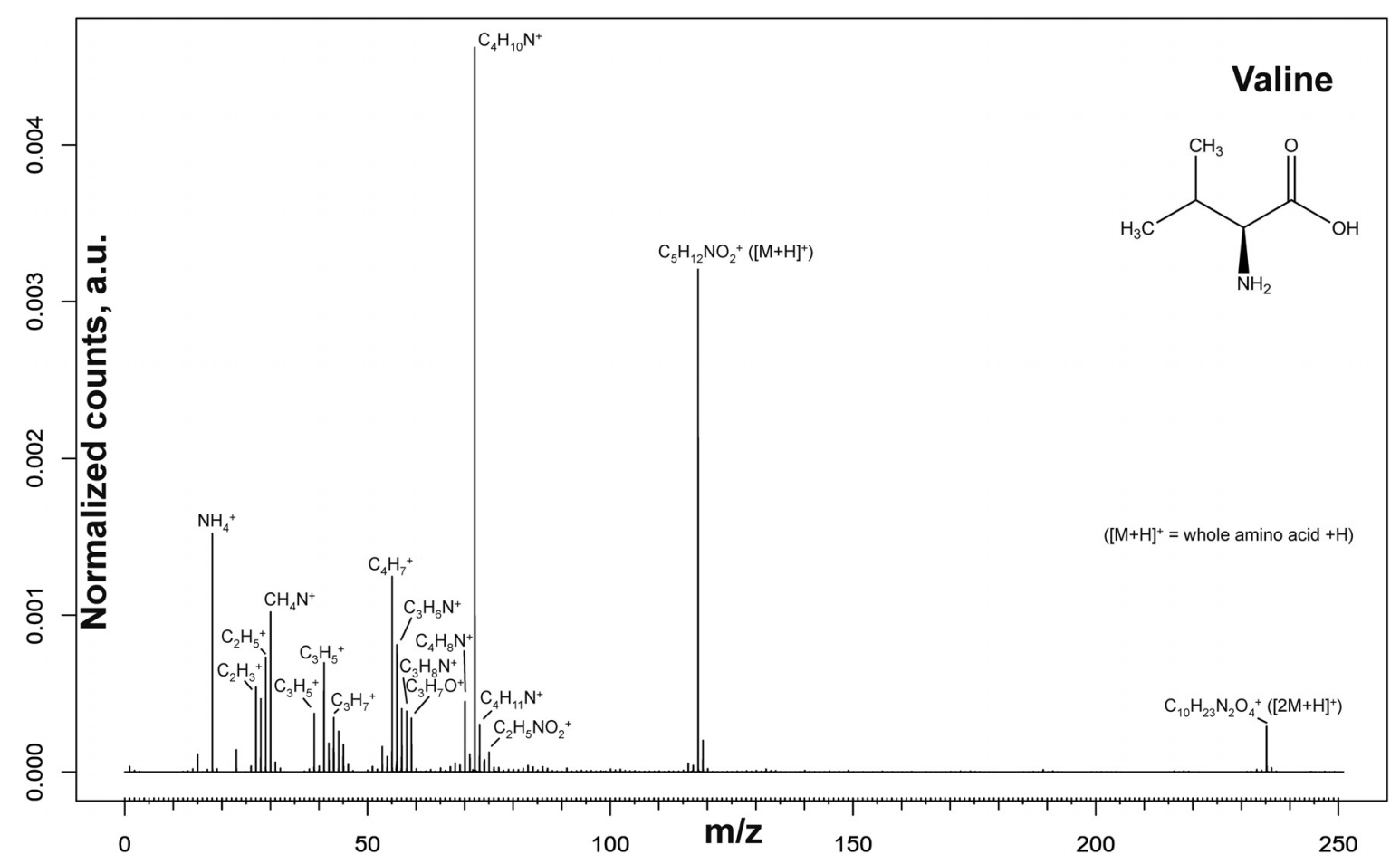

\begin{tabular}{rc}
\hline Accession \# & 01474-01, 01474-02, 01474-03, 01474-04 \\
\hline Host Material & Silicon wafer \\
Technique & SIMS \\
Secondary Source Polarity & Positive \\
Mass Range Minimum & $0 \mathrm{Da}$ \\
Mass Range Maximum & $250 \mathrm{Da}$ \\
Species Used for Mass Calibration & $\mathrm{CH}_{3}{ }^{+}, \mathrm{NH}_{3}{ }^{+}, \mathrm{NH}_{4}{ }^{+}, \mathrm{CH}_{2} \mathrm{~N}^{+}, \mathrm{C}_{5} \mathrm{H}_{13} \mathrm{NO}_{2}{ }^{+}$ \\
Primary lon Dose & $4.73 \times 10^{11} \mathrm{~cm}^{-2}$ \\
Primary Species & $\mathrm{Bi}_{3}{ }^{+}$ \\
Primary lon Pulse Width & $29.3 \mathrm{~ns}^{-}$ \\
Pulsed Beam Current & $0.00037 \mathrm{nA}^{2}$ \\
Beam Raster Size & $200 \times 200 \mu \mathrm{m}{ }^{2}$ \\
\hline
\end{tabular}



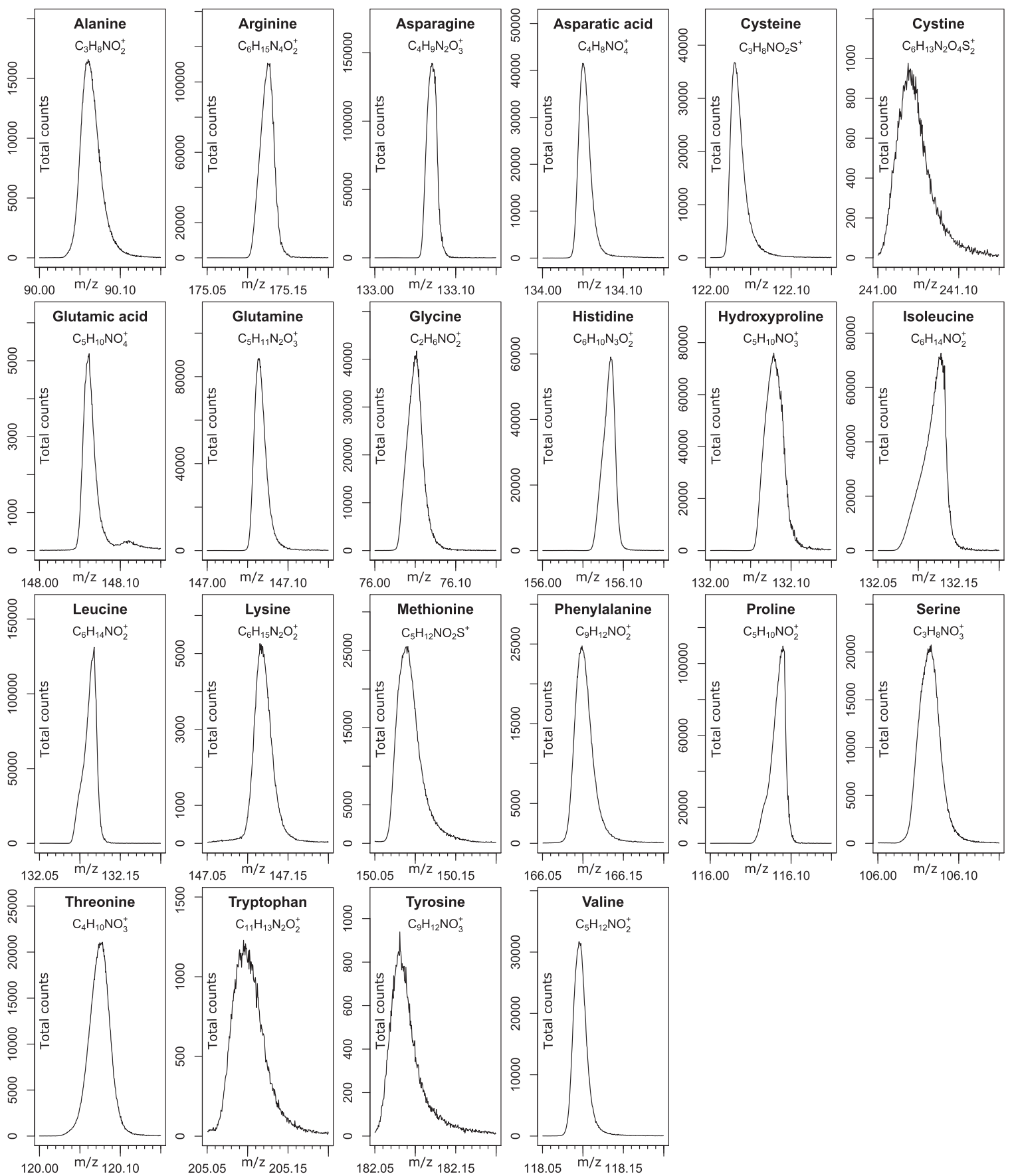

01453-01, 01454-01, 01455-01, 01456-01, 01457-01, 01458-01, 01459-01, 01460-01, 01461-01, 01462-01, 01463-01, 01464-01, 01465-01, 01465-01, 01467-01, 01468-01, 01469-01, 01470-01, 01471-01, 01472-01, 


\begin{tabular}{|c|c|c|c|}
\hline \multicolumn{4}{|c|}{ SPECTRAL FEATURES TABLE } \\
\hline Mass (Da) & Peak assignment & Peak present in following amino acids: & Peak most prominent in: \\
\hline 17.0260 & $\mathrm{NH}_{3}^{+}$ & all amino acids & - \\
\hline 18.0338 & $\mathrm{NH}_{4}^{+}$ & all amino acids & - \\
\hline 28.0182 & $\mathrm{CH}_{2} \mathrm{~N}^{+}$ & all amino acids & - \\
\hline 30.0338 & $\mathrm{CH}_{4} \mathrm{~N}^{+}$ & all amino acids & - \\
\hline 31.9715 & $\mathrm{~S}^{+}$ & cysteine, cystine, methionine & cysteine, cystine, methionine \\
\hline 34.9950 & $\mathrm{SH}_{3}^{+}$ & cysteine, cystine, methionine & cysteine, cystine, methionine \\
\hline 39.0229 & $\mathrm{C}_{3} \mathrm{H}_{3}{ }^{+}$ & all amino acids except for glycine & - \\
\hline 41.0386 & $\mathrm{C}_{3} \mathrm{H}_{5}^{+}$ & all amino acids except for glycine & - \\
\hline 42.0338 & $\mathrm{C}_{2} \mathrm{H}_{4} \mathrm{~N}^{+}$ & all amino acids & - \\
\hline 43.0417 & $\mathrm{C}_{2} \mathrm{H}_{5} \mathrm{~N}^{+}$ & all amino acids & - \\
\hline 43.0542 & $\mathrm{C}_{3} \mathrm{H}_{7}^{+}$ & most amino acids & - \\
\hline 43.0718 & $\mathrm{C}_{2} \mathrm{H}_{3} \mathrm{O}^{+}$ & all amino acids & - \\
\hline 44.0495 & $\mathrm{C}_{2} \mathrm{H}_{6} \mathrm{~N}^{+}$ & all amino acids & - \\
\hline 44.9793 & $\mathrm{CHS}^{+}$ & cysteine, cystine, methionine & cysteine, cystine, methionine \\
\hline 45.0335 & $\mathrm{C}_{2} \mathrm{H}_{5} \mathrm{O}^{+}$ & all amino acids & - \\
\hline 45.0573 & $\mathrm{C}_{2} \mathrm{H}_{7} \mathrm{~N}^{+}$ & all amino acids & - \\
\hline 45.9872 & $\mathrm{CH}_{2} \mathrm{~S}^{+}$ & cysteine, cystine, methionine & cysteine, cystine, methionine \\
\hline 46.0651 & $\mathrm{C}_{2} \mathrm{H}_{8} \mathrm{~N}^{+}$ & all amino acids & - \\
\hline 46.9950 & $\mathrm{CH}_{3} \mathrm{~S}^{+}$ & cysteine, cystine, methionine & cysteine, cystine, methionine \\
\hline 47.0491 & $\mathrm{C}_{2} \mathrm{H}_{7} \mathrm{O}^{+}$ & all amino acids & - \\
\hline 51.0229 & $\mathrm{C}_{4} \mathrm{H}_{3}{ }^{+}$ & multiple amino acids & - \\
\hline 53.0386 & $\mathrm{C}_{4} \mathrm{H}_{5}{ }^{+}$ & multiple amino acids & - \\
\hline 55.0178 & $\mathrm{C}_{3} \mathrm{H}_{3} \mathrm{O}^{+}$ & all amino acids except for glycine & - \\
\hline 55.0542 & $\mathrm{C}_{4} \mathrm{H}_{7}^{+}$ & multiple amino acids & - \\
\hline 56.0495 & $\mathrm{C}_{3} \mathrm{H}_{6} \mathrm{~N}^{+}$ & all amino acids except for glycine & - \\
\hline 56.0621 & $\mathrm{C}_{4} \mathrm{H}_{8}^{+}$ & multiple amino acids & - \\
\hline 57.0335 & $\mathrm{C}_{3} \mathrm{H}_{5} \mathrm{O}^{+}$ & all amino acids except for glycine & - \\
\hline 57.0573 & $\mathrm{C}_{3} \mathrm{H}_{7} \mathrm{~N}^{+}$ & multiple amino acids & - \\
\hline 58.0287 & $\mathrm{C}_{2} \mathrm{H}_{4} \mathrm{NO}^{+}$ & all amino acids & - \\
\hline 58.0651 & $\mathrm{C}_{3} \mathrm{H}_{8} \mathrm{~N}^{+}$ & all amino acids except for glycine & - \\
\hline 58.0777 & $\mathrm{C}_{4} \mathrm{H}_{10}^{+}$ & multiple amino acids & - \\
\hline 58.9950 & $\mathrm{C}_{2} \mathrm{H}_{3} \mathrm{~S}^{+}$ & cysteine, cystine, methionine & cysteine, cystine, methionine \\
\hline 59.0491 & $\mathrm{C}_{3} \mathrm{H}_{7} \mathrm{O}^{+}$ & all amino acids except for glycine & - \\
\hline 60.0444 & $\mathrm{C}_{2} \mathrm{H}_{6} \mathrm{NO}^{+}$ & all amino acids & - \\
\hline 60.0556 & $\mathrm{CH}_{6} \mathrm{~N}_{3}^{+}$ & arginine & arginine \\
\hline 61.0106 & $\mathrm{C}_{2} \mathrm{H}_{5} \mathrm{~S}^{+}$ & cysteine, cystine, methionine & cysteine, cystine, methionine \\
\hline 61.0284 & $\mathrm{C}_{2} \mathrm{H}_{5} \mathrm{O}_{2}^{+}$ & all amino acids & - \\
\hline 63.9436 & $\mathrm{~S}_{2}{ }^{+}$ & cystine & cystine \\
\hline 67.0542 & $\mathrm{C}_{5} \mathrm{H}_{7}^{+}$ & multiple amino acids & - \\
\hline 68.0495 & $\mathrm{C}_{4} \mathrm{H}_{6} \mathrm{~N}^{+}$ & multiple amino acids & - \\
\hline 68.0621 & $\mathrm{C}_{5} \mathrm{H}_{8}^{+}$ & multiple amino acids & - \\
\hline 69.0335 & $\mathrm{C}_{4} \mathrm{H}_{5} \mathrm{O}^{+}$ & multiple amino acids & - \\
\hline 69.0447 & $\mathrm{C}_{3} \mathrm{H}_{5} \mathrm{~N}_{2}^{+}$ & arginine, asparagine, histidine & asparagine, histidine \\
\hline 69.0573 & $\mathrm{C}_{4} \mathrm{H}_{7} \mathrm{~N}^{+}$ & multiple amino acids & - \\
\hline 69.0699 & $\mathrm{C}_{5} \mathrm{H}_{9}{ }^{+}$ & multiple amino acids & - \\
\hline 70.0287 & $\mathrm{C}_{3} \mathrm{H}_{4} \mathrm{NO}^{+}$ & all amino acids except for glycine & - \\
\hline
\end{tabular}




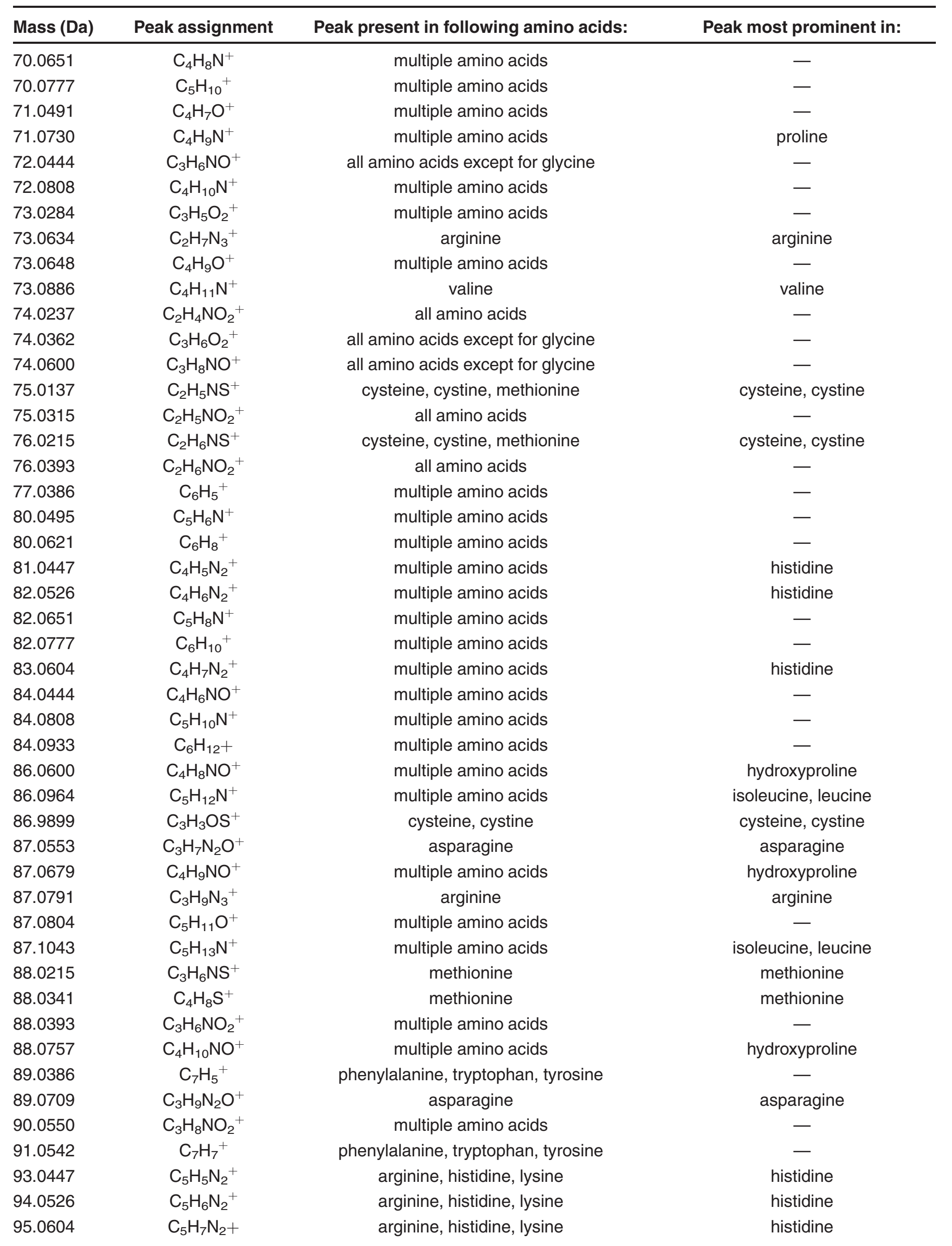




\begin{tabular}{|c|c|c|c|}
\hline Mass (Da) & Peak assignment & Peak present in following amino acids: & Peak most prominent in: \\
\hline 96.0808 & $\mathrm{C}_{6} \mathrm{H}_{10} \mathrm{~N}^{+}$ & isoleucine, leucine, lysine & - \\
\hline 98.0964 & $\mathrm{C}_{6} \mathrm{H}_{12} \mathrm{~N}^{+}$ & isoleucine, leucine, lysine & - \\
\hline 100.0393 & $\mathrm{C}_{4} \mathrm{H}_{6} \mathrm{NO}_{2}^{+}$ & multiple amino acids & - \\
\hline 100.0869 & $\mathrm{C}_{4} \mathrm{H}_{10} \mathrm{~N}_{3}^{+}$ & arginine & arginine \\
\hline 101.0709 & $\mathrm{C}_{4} \mathrm{H}_{9} \mathrm{~N}_{2} \mathrm{O}^{+}$ & asparagine, glutamine & glutamine \\
\hline 102.0464 & $\mathrm{C}_{8} \mathrm{H}_{6}{ }^{+}$ & phenylalanine, tryptophan, tyrosine & - \\
\hline 102.0550 & $\mathrm{C}_{4} \mathrm{H}_{8} \mathrm{NO}_{2}^{+}$ & multiple amino acids & - \\
\hline 103.0542 & $\mathrm{C}_{8} \mathrm{H}_{7}^{+}$ & phenylalanine, tryptophan, tyrosine & phenylalanine \\
\hline 103.0866 & $\mathrm{C}_{4} \mathrm{H}_{11} \mathrm{~N}_{2} \mathrm{O}^{+}$ & asparagine, glutamine & glutamine \\
\hline 104.0528 & $\mathrm{C}_{4} \mathrm{H}_{10} \mathrm{NS}^{+}$ & methionine & methionine \\
\hline 105.0005 & $\mathrm{C}_{3} \mathrm{H}_{5} \mathrm{O}_{2} \mathrm{~S}^{+}$ & cysteine, dystine & cysteine, cystine \\
\hline 105.0699 & $\mathrm{C}_{8} \mathrm{H}_{9}^{+}$ & phenylalanine, tyrosine & phenylalanine \\
\hline 106.0499 & $\mathrm{C}_{3} \mathrm{H}_{8} \mathrm{NO}_{3}{ }^{+}$ & serine, threonine & serine \\
\hline 107.0491 & $\mathrm{C}_{7} \mathrm{H}_{7} \mathrm{O}^{+}$ & phenylalanine, tyrosine & - \\
\hline 107.0577 & $\mathrm{C}_{3} \mathrm{H}_{9} \mathrm{NO}_{3}^{+}$ & hydroxyproline, serine, threonine & serine \\
\hline 110.0713 & $\mathrm{C}_{5} \mathrm{H}_{8} \mathrm{~N}_{3}^{+}$ & arginine, histidine & histidine \\
\hline 110.0839 & $\mathrm{C}_{6} \mathrm{H}_{10} \mathrm{~N}_{2}^{+}$ & lysine & lysine \\
\hline 112.0869 & $\mathrm{C}_{5} \mathrm{H}_{10} \mathrm{~N}_{3}^{+}$ & arginine & arginine \\
\hline 114.0550 & $\mathrm{C}_{5} \mathrm{H}_{8} \mathrm{NO}_{2}^{+}$ & multiple amino acids & - \\
\hline 114.1026 & $\mathrm{C}_{5} \mathrm{H}_{12} \mathrm{~N}_{3}^{+}$ & arginine & arginine \\
\hline 115.0417 & $\mathrm{C}_{8} \mathrm{H}_{5} \mathrm{~N}^{+}$ & phenylalanine, tryptophan, tyrosine & - \\
\hline 115.0628 & $\mathrm{C}_{5} \mathrm{H}_{9} \mathrm{NO}_{2}^{+}$ & multiple amino acids & - \\
\hline 116.0342 & $\mathrm{C}_{4} \mathrm{H}_{6} \mathrm{NO}_{3}^{+}$ & asparagine & asparagine \\
\hline 116.0706 & $\mathrm{C}_{5} \mathrm{H}_{10} \mathrm{NO}_{2}^{+}$ & multiple amino acids & proline \\
\hline 117.0573 & $\mathrm{C}_{8} \mathrm{H}_{7} \mathrm{~N}^{+}$ & phenylalanine, tryptophan, tyrosine & - \\
\hline 118.0651 & $\mathrm{C}_{8} \mathrm{H}_{8} \mathrm{~N}^{+}$ & phenylalanine, tryptophan, tyrosine & phenylalanine, tryptophan \\
\hline 118.0862 & $\mathrm{C}_{5} \mathrm{H}_{12} \mathrm{NO}_{2}^{+}$ & multiple amino acids & asparatic acid, glutamic acid, valine \\
\hline 119.0491 & $\mathrm{C}_{8} \mathrm{H}_{7} \mathrm{O}^{+}$ & phenylalanine, tryptophan, tyrosine & - \\
\hline 120.0114 & $\mathrm{C}_{3} \mathrm{H}_{6} \mathrm{NO}_{2} \mathrm{~S}^{+}$ & cysteine, cystine & cysteine, cystine \\
\hline 120.0444 & $\mathrm{C}_{7} \mathrm{H}_{6} \mathrm{NO}^{+}$ & tryptophan, tyrosine & tryptophan \\
\hline 120.0655 & $\mathrm{C}_{4} \mathrm{H}_{10} \mathrm{NO}_{3}^{+}$ & threonine & threonine \\
\hline 120.0808 & $\mathrm{C}_{8} \mathrm{H}_{10} \mathrm{~N}^{+}$ & phenylalanine & phenylalanine \\
\hline 121.0648 & $\mathrm{C}_{8} \mathrm{H}_{9} \mathrm{O}^{+}$ & phenylalanine, tryptophan, tyrosine & - \\
\hline 121.0886 & $\mathrm{C}_{8} \mathrm{H}_{11} \mathrm{~N}^{+}$ & phenylalanine & phenylalanine \\
\hline 122.0270 & $\mathrm{C}_{3} \mathrm{H}_{8} \mathrm{NO}_{2} \mathrm{~S}^{+}$ & cysteine, cystine & cysteine, cystine \\
\hline 128.0706 & $\mathrm{C}_{6} \mathrm{H}_{10} \mathrm{NO}_{2}^{+}$ & isoleucine, leucine, lysine & isoleucine, leucine, lysine \\
\hline 129.0420 & $\mathrm{C}_{5} \mathrm{H}_{7} \mathrm{NO}_{3}^{+}$ & glutamic acid, glutamine, hydroxyproline & glutamine \\
\hline 129.1135 & $\mathrm{C}_{5} \mathrm{H}_{13} \mathrm{~N}_{4}^{+}$ & arginine & arginine \\
\hline 130.0499 & $\mathrm{C}_{5} \mathrm{H}_{8} \mathrm{NO}_{3}^{+}$ & glutamic acid, glutamine, hydroxyproline & glutamine \\
\hline 130.0651 & $\mathrm{C}_{9} \mathrm{H}_{8} \mathrm{~N}^{+}$ & phenylalanine, tryptophan, tyrosine & tryptophan \\
\hline 130.1213 & $\mathrm{C}_{5} \mathrm{H}_{14} \mathrm{~N}_{4}^{+}$ & arginine & arginine \\
\hline 131.0730 & $\mathrm{C}_{9} \mathrm{H}_{9} \mathrm{~N}^{+}$ & phenylalanine, tryptophan, tyrosine & tryptophan \\
\hline 131.1291 & $\mathrm{C}_{5} \mathrm{H}_{15} \mathrm{~N}_{4}^{+}$ & arginine & arginine \\
\hline 132.0655 & $\mathrm{C}_{5} \mathrm{H}_{10} \mathrm{NO}_{3}^{+}$ & glutamic acid, glutamine, hydroxyproline & hydroxyproline \\
\hline 132.0808 & $\mathrm{C}_{9} \mathrm{H}_{10} \mathrm{~N}^{+}$ & phenylalanine, tryptophan, tyrosine & tryptophan \\
\hline 132.1019 & $\mathrm{C}_{6} \mathrm{H}_{14} \mathrm{NO}_{2}^{+}$ & isoleucine, leucine, lysine & isoleucine, leucine \\
\hline 133.0318 & $\mathrm{C}_{5} \mathrm{H}_{9} \mathrm{O}_{2} \mathrm{~S}^{+}$ & methionine & methionine \\
\hline
\end{tabular}




\begin{tabular}{|c|c|c|c|}
\hline Mass (Da) & Peak assignment & Peak present in following amino acids: & Peak most prominent in: \\
\hline 133.0608 & $\mathrm{C}_{4} \mathrm{H}_{9} \mathrm{~N}_{2} \mathrm{O}_{3}^{+}$ & asparagine & asparagine \\
\hline 134.0448 & $\mathrm{C}_{4} \mathrm{H}_{8} \mathrm{NO}_{4}^{+}$ & asparatic acid & asparatic acid \\
\hline 136.0757 & $\mathrm{C}_{8} \mathrm{H}_{10} \mathrm{NO}^{+}$ & tyrosine & tyrosine \\
\hline 143.0730 & $\mathrm{C}_{10} \mathrm{H}_{9} \mathrm{~N}^{+}$ & tryptophan & tryptophan \\
\hline 144.0682 & $\mathrm{C}_{9} \mathrm{H}_{8} \mathrm{~N}_{2}^{+}$ & tryptophan & tryptophan \\
\hline 147.0764 & $\mathrm{C}_{5} \mathrm{H}_{11} \mathrm{~N}_{2} \mathrm{O}_{3}{ }^{+}$ & glutamine & glutamine \\
\hline 147.1128 & $\mathrm{C}_{6} \mathrm{H}_{15} \mathrm{~N}_{2} \mathrm{O}_{2}^{+}$ & lysine & lysine \\
\hline 148.0604 & $\mathrm{C}_{5} \mathrm{H}_{10} \mathrm{NO}_{4}^{+}$ & glutamic acid & glutamic acid \\
\hline 149.0107 & $\mathrm{C}_{7} \mathrm{H}_{3} \mathrm{NO}_{3}^{+}$ & tyrosine & tyrosine \\
\hline 150.0583 & $\mathrm{C}_{5} \mathrm{H}_{12} \mathrm{NO}_{2} \mathrm{~S}^{+}$ & methionine & methionine \\
\hline 151.9834 & $\mathrm{C}_{3} \mathrm{H}_{6} \mathrm{NO}_{2} \mathrm{~S}_{2}^{+}$ & cystine & cystine \\
\hline 156.0768 & $\mathrm{C}_{6} \mathrm{H}_{10} \mathrm{~N}_{3} \mathrm{O}_{2}^{+}$ & histidine & histidine \\
\hline 157.0760 & $\mathrm{C}_{10} \mathrm{H}_{9} \mathrm{~N}_{2}^{+}$ & tryptophan & tryptophan \\
\hline 158.0839 & $\mathrm{C}_{10} \mathrm{H}_{10} \mathrm{~N}_{2}^{+}$ & tryptophan & tryptophan \\
\hline 160.1081 & $\mathrm{C}_{6} \mathrm{H}_{14} \mathrm{~N}_{3} \mathrm{O}_{2}{ }^{+}$ & arginine & arginine \\
\hline 165.0546 & $\mathrm{C}_{9} \mathrm{H}_{9} \mathrm{O}_{3}^{+}$ & tyrosine & tyrosine \\
\hline 165.0764 & $\mathrm{C}_{9} \mathrm{H}_{11} \mathrm{NO}_{2}{ }^{+}$ & tyrosine & tyrosine \\
\hline 166.0863 & $\mathrm{C}_{9} \mathrm{H}_{12} \mathrm{NO}_{2}^{+}$ & phenylalanine & phenylalanine \\
\hline 169.0917 & $\mathrm{C}_{10} \mathrm{H}_{11} \mathrm{~N}_{2}^{+}$ & tryptophan & tryptophan \\
\hline 175.1190 & $\mathrm{C}_{6} \mathrm{H}_{15} \mathrm{~N}_{4} \mathrm{O}_{2}^{+}$ & arginine & arginine \\
\hline 182.0812 & $\mathrm{C}_{9} \mathrm{H}_{12} \mathrm{NO}_{3}^{+}$ & tyrosine & tyrosine \\
\hline 188.0944 & $\mathrm{C}_{11} \mathrm{H}_{12} \mathrm{~N}_{2} \mathrm{O}^{+}$ & tryptophan & tryptophan \\
\hline 195.0256 & $\mathrm{C}_{5} \mathrm{H}_{11} \mathrm{~N}_{2} \mathrm{O}_{2} \mathrm{~S}_{2}^{+}$ & cystine & cystine \\
\hline 205.0972 & $\mathrm{C}_{11} \mathrm{H}_{13} \mathrm{~N}_{2} \mathrm{O}_{2}+$ & tryptophan & tryptophan \\
\hline 207.0256 & $\mathrm{C}_{6} \mathrm{H}_{11} \mathrm{~N}_{2} \mathrm{O}_{2} \mathrm{~S}_{2}^{+}$ & cystine & cystine \\
\hline 241.0311 & $\mathrm{C}_{6} \mathrm{H}_{13} \mathrm{~N}_{2} \mathrm{O}_{4} \mathrm{~S}_{2}^{+}$ & cystine & cystine \\
\hline
\end{tabular}

